TRANSACTIONS OF THE

AMERICAN MATHEMATICAL SOCIETY

Volume 353, Number 2, Pages 491-518

S 0002-9947(00)02659-3

Article electronically published on September 21, 2000

\title{
THE COMPLETENESS OF THE ISOMORPHISM RELATION FOR COUNTABLE BOOLEAN ALGEBRAS
}

\author{
RICCARDO CAMERLO AND SU GAO
}

\begin{abstract}
We show that the isomorphism relation for countable Boolean algebras is Borel complete, i.e., the isomorphism relation for arbitrary countable structures is Borel reducible to that for countable Boolean algebras. This implies that Ketonen's classification of countable Boolean algebras is optimal in the sense that the kind of objects used for the complete invariants cannot be improved in an essential way. We also give a stronger form of the Vaught conjecture for Boolean algebras which states that, for any complete first-order theory of Boolean algebras that has more than one countable model up to isomorphism, the class of countable models for the theory is Borel complete. The results are applied to settle many other classification problems related to countable Boolean algebras and separable Boolean spaces. In particular, we will show that the following equivalence relations are Borel complete: the translation equivalence between closed subsets of the Cantor space, the isomorphism relation between ideals of the countable atomless Boolean algebra, the conjugacy equivalence of the autohomeomorphisms of the Cantor space, etc. Another corollary of our results is the Borel completeness of the commutative $\mathrm{AF} C^{*}$-algebras, which in turn gives rise to similar results for Bratteli diagrams and dimension groups.
\end{abstract}

\section{INTRODUCTION}

In this paper we address the question: how complicated is the classification problem for countable Boolean algebras? Some classes of countable Boolean algebras admit a very simple classification. For example, the class of countable superatomic Boolean algebras has complete invariants of the form $(\alpha, n)$, where $\alpha$ is a countable ordinal and $n$ is a natural number. (For a proof, see [Ko89, Theorem 17.1].) The situation is different when we consider the class of all countable Boolean algebras. A complete classification for countable Boolean algebras was given by Ketonen ([Ket78] ) and was used by him to establish some interesting results about the structure of the monoid of isomorphism types of countable Boolean algebras. However, the Ketonen invariants are very complicated, and it is conceivable that if one could carry out a classification using a simpler kind of invariants, then Ketonen's results about the monoid of isomorphism types could be improved. Let us briefly summarize Ketonen's approach to the classification problem. This was done in two steps. First, an arbitrary countable Boolean algebra is decomposed into a superatomic part and a uniform part. It turns out that the uniform part has a

Received by the editors March 11, 1999.

2000 Mathematics Subject Classification. Primary 03E15, 06E15.

Key words and phrases. Borel reducibility, polish group actions, definable equivalence relations, separable Boolean spaces. 
complete invariant in the form of an additive function $\sigma: B_{\eta} \rightarrow \omega_{1}$, where $B_{\eta}$ is the (unique) countable atomless Boolean algebra, modulo an equivalence induced by automorphisms of $B_{\eta}$. In the second step, the classification is completed by assigning to each such additive function a hereditarily countable structure as its complete invariant. (A complete account of the classification can also be found in Pi89.)

The fact that countable Boolean algebras admit hereditarily countable structures as their complete invariants is hardly surprising. It has been known to logicians for a long time that countable structures in any countable language admit a classification using hereditarily countable sets as complete invariants. Specifically, the Scott analysis (cf. Ba75]) provides such an abstract classification for any countable structure, if we understand the Scott sentences as codes for certain hereditarily countable sets. Therefore, in terms of the kind of objects used as complete invariants, Ketonen's classification offers no essential improvement to the classification problem for countable Boolean algebras (nevertheless, it is suitable for some other purposes).

What kind of objects would be considered an essential improvement to hereditarily countable sets? One candidate is the invariants used by Ulm to classify countable torsion abelian groups, which are essentially countable sequences of countable ordinals (cf. [HK95]). The frustrating situation with the classification of countable Boolean algebras suggests that there might not be an Ulm type classification for countable Boolean algebras. We can formulate statements of this sort in the framework of the study of definable equivalence relations, as carried out in, e.g., [BK96], [FS89] and [Hj98.

The central concept in the study of definable equivalence relations is the notion of Borel reducibility. For standard Borel spaces $X, Y$ and equivalence relations $E$ on $X$ and $F$ on $Y$, we say that $E$ is Borel reducible to $F$, denoted by $E \leq_{B} F$, if there is a Borel function $f: X \rightarrow Y$ such that, for any $x, y \in X$,

$$
x E y \Leftrightarrow f(x) F f(y) .
$$

If $E \leq_{B} F$ and $F \leq_{B} E$, then we say that $E$ and $F$ are Borel equivalent and write $E \sim_{B} F$. The isomorphism relation for countable structures fits in this category of equivalence relations.

Let $\mathcal{L}$ be an arbitrary countable language and $\operatorname{Mod}(\mathcal{L})$ be the space of all countable models of $\mathcal{L}$ with domain $\mathbb{N}$. $\operatorname{Mod}(\mathcal{L})$ is a standard Borel space. Let $T$ be a countable theory in $\mathcal{L}_{\omega_{1} \omega}$. Then $\operatorname{Mod}(T)$, the space of all models of $T$ with domain $\mathbb{N}$, is a Borel subspace of $\operatorname{Mod}(\mathcal{L})$, hence also a standard Borel space. The isomorphism relation for models of $T$ can be viewed as an orbit equivalence relation induced by a Borel action of $S_{\infty}$ on $\operatorname{Mod}(T)$, where $S_{\infty}$ is the Polish group of all permutations of $\mathbb{N}$ (cf., Kec95, 16.C]).

By results of BK96, there is an orbit equivalence relation $C_{\infty}$ induced by a Borel action of $S_{\infty}$ which is complete in the sense that, for any closed subgroup $G$ of $S_{\infty}$ and any orbit equivalence relation $E_{G}$ induced by a Borel action of $G$ (on some standard Borel space), we always have that $E_{G} \leq_{B} C_{\infty}$. In general, we say that an equivalence relation is Borel complete if it is Borel equivalent to $C_{\infty}$. If $E$ is the isomorphism relation for models of a theory $T$, then $E \leq_{B} C_{\infty}$ by observations in the preceding paragraph. Therefore $E$ is Borel complete if and only if $C_{\infty} \leq_{B} E$. By results of [FS89] and BK96, this is also equivalent to the statement that, for 
any countable language $\mathcal{L}$, the isomorphism relation on $\operatorname{Mod}(\mathcal{L})$ is Borel reducible to $E$.

For two invariant Borel classes of countable models $\mathcal{A}$ and $\mathcal{B}$, we say that $\mathcal{A}$ is Borel reducible to $\mathcal{B}$ and write $\mathcal{A} \leq_{B} \mathcal{B}$ iff the isomorphism relation on $\mathcal{A}$ is Borel reducible to that on $\mathcal{B}$. When the isomorphism on $\mathcal{A}$ is Borel complete, we say that $\mathcal{A}$ is Borel complete, or that $\mathcal{A}$ has completely many countable models (up to isomorphism).

Classical results in model theory imply that the classes of all countable graphs, directed graphs, groups, lattices, etc. are each Borel complete (cf. [Ho93]). In [FS89] it is established that countable trees, linear orderings, and fields of characteristic $p$ for any prime $p$ or $p=0$ have also Borel complete isomorphism relations. On the other hand, it is shown that the isomorphism relation for countable torsion abelian groups is not Borel complete. It is also known that if the invariant Borel class $\mathcal{A}$ of countable models is Borel complete, then $\mathcal{A}$ does not admit an Ulm type classification. [HK95] contains a detailed treatment of Ulm type classification.

The first major theorem that we prove in this paper is the following.

Theorem. The class of all countable Boolean algebras is Borel complete.

This has a topological counterpart.

Theorem. The homeomorphism relation between separable Boolean spaces, i.e., zero-dimensional compact metrizable spaces, is Borel complete.

In particular, these imply that there is no Ulm type classification for countable Boolean algebras, confirming our previous speculation. Furthermore, these theorems suggest that the objects used as complete invariants in any classification of countable Boolean algebras cannot be essentially simpler than hereditarily countable sets. In this sense, Ketonen's classification is optimal.

Our strategy for the proof of the main theorems is to define a reduction from countable graphs to separable Boolean spaces. In section 2 we will first justify this approach by unravelling the effectiveness of the Stone duality.

Our next major result is a strengthening of the main theorem about Boolean algebras to a very strong form of the Vaught conjecture. The original Vaught conjecture states that any complete consistent first-order theory either has countably many countable models up to isomorphism, or else has $2^{\aleph_{0}}$ many. The Vaught conjecture for Boolean algebras has been verified by Iverson ([Iv91] $)$. In fact, Iverson's result is already a strong form of the conjecture. Continuing in this direction, we go one step further by proving the following theorem.

Theorem (A very strong Vaught conjecture). Let $T$ be a complete consistent firstorder theory of Boolean algebras. Then either T has only one countable model up to isomorphism or else $\operatorname{Mod}(T)$ is Borel complete.

This theorem is a simultaneous strengthening of Iverson's theorem and our main theorem. The proof will be presented in section 4 .

In section 5 we consider various other definable equivalence relations related to countable Boolean algebras or separable Boolean spaces. Among the ones we prove Borel complete, are the translation relation between closed subsets of the Cantor space, the isomorphism relation between ideals of the countable atomless Boolean algebra, the conjugacy equivalence relation of the automorphisms of the countable atomless Boolean algebra (or the autohomeomorphisms of the Cantor 
space), etc. We also prove some further results about Ketonen's classification. Specifically, we show that the decomposition of uniform Boolean algebras into $\aleph_{1}$ many classes according to the heights of their corresponding additive functions does not essentially simplify the classification problem. This is done by proving that each class is still Borel complete. In the following we summarize the most important results. The formal definitions and rigorous statements of the results are postponed to section 5 since some equivalence relations need to be defined and explained in more detail.

Theorem. The following equivalence relations are Borel complete, where $B_{\eta}$ is the countable atomless Boolean algebra:

(a) the translation relation between closed subsets of the Cantor space, i.e., two such sets are equivalent iff there is an autohomeomorphism of the Cantor space translating one to the other;

(b) the conjugacy equivalence relation between autohomeomorphisms of the Cantor space;

(c) the isomorphism relation between pairs $\left(B_{\eta}, I\right)$, where $I$ is an ideal of $B_{\eta}$;

(d) the isomorphism relation between pairs $\left(B_{\eta}, \varphi\right)$, where $\varphi$ is an automorphism of $B_{\eta}$.

Theorem. For each countable ordinal $\alpha>0$, the class of all uniform countable Boolean algebras of height $\alpha$ is Borel complete.

In section 6 we present some applications of our work on Boolean algebras to the study of $C^{*}$-algebras. We establish the Borel completeness of $\mathrm{AF} C^{*}$-algebras, Bratteli diagrams and dimension groups. In particular, we consider the class of commutative $\mathrm{AF} C^{*}$-algebras, which is presumably the simplest kind of $C^{*}$-algebras, and prove their Borel completeness.

Theorem. The following equivalence relations are Borel complete:

(a) the isomorphism relation between commutative $\mathrm{AF} C^{*}$-algebras;

(b) the relation of equivalence between Bratteli diagrams;

(c) the isomorphism relation between dimension groups.

This confirms the intuition of many $C^{*}$-algebraists that interesting results about general $C^{*}$-algebras may be already hidden in the study of simpler classes. Below let us emphasize this principle by stating a corollary which only mentions concepts in the category of $C^{*}$-algebras.

Theorem. There is a Borel reduction from all $\mathrm{AF} C^{*}$-algebras to the commutative ones, i.e, an assignment $\theta$ which associates to each $\mathrm{AF} C^{*}$-algebra $A$ a commutative $\mathrm{AF}$ algebra $\theta(A)$ such that

$$
A \cong A^{\prime} \Leftrightarrow \theta(A) \cong \theta\left(A^{\prime}\right) .
$$

Finally in section 7 we make some further remarks about the classifications of countable Boolean algebras or general countable structures. Some open problems are stated and explained.

\section{Some PRELIMINARIES}

In this section we recall some basic concepts and results about Boolean algebras and fix some notation for use in the rest of the paper. Since we are going to deal with equivalence relations arising from different fields of mathematics, the main 
effort here is to clarify that (i) the underlying spaces for the equivalence relations under consideration are all standard Borel spaces, or even Polish spaces, and (ii) the equivalence relations are all strongly definable in the sense that they are either orbit equivalence relations induced by Borel actions of Polish groups, or Borel reducible to such. First of all, we give some remarks on this aspect of the Stone duality. In section 3 we will use Stone duality without further comment. This kind of clarification will be resumed in section 5 for other equivalence relations.

I. The Stone duality.

For an account of Stone duality see, e.g., Ko89. Our terminology for the topological concepts follows [Kec95].

Let $\mathcal{L}$ be the language for Boolean algebras, $T_{\mathrm{BA}}$ be the axioms of Boolean algebras in the language $\mathcal{L}$ and let $\mathrm{CBA}=\operatorname{Mod}\left(T_{\mathrm{BA}}\right)$ be the space of models of $T_{\mathrm{BA}}$ with universe $\mathbb{N}$. Then CBA is a standard Borel space and the isomorphism relation $\cong$ on $\mathrm{CBA}$ is the orbit equivalence relation of a Borel $S_{\infty}$ action, as noted in section 1 .

Let $2^{\mathbb{N}}$ be the Cantor space and $\mathcal{K}\left(2^{\mathbb{N}}\right)$ be the space of all closed (compact) subsets of $2^{\mathbb{N}}$, equipped with the Vietoris topology. $\mathcal{K}\left(2^{\mathbb{N}}\right)$ is compact metrizable, therefore Polish. We denote also by $\cong$ the homeomorphism relation on $\mathcal{K}\left(2^{\mathbb{N}}\right)$.

We will need a form of the Stone duality which states that there are Borel functions $\varphi: \mathrm{CBA} \rightarrow \mathcal{K}\left(2^{\mathbb{N}}\right)$ and $\psi: \mathcal{K}\left(2^{\mathbb{N}}\right) \rightarrow$ CBA such that (a) for any $B, B^{\prime} \in$ $\mathrm{CBA}, B \cong B^{\prime}$ iff $\varphi(B) \cong \varphi\left(B^{\prime}\right)$, and (b) for any $K, K^{\prime} \in \mathcal{K}\left(2^{\mathbb{N}}\right), K \cong K^{\prime}$ iff $\psi(K) \cong$ $\psi\left(K^{\prime}\right)$; in other words, the isomorphism relation on CBA is Borel equivalent to the homeomorphism relation on $\mathcal{K}\left(2^{\mathbb{N}}\right)$. We use the functions $\varphi$ and $\psi$, where $\varphi(B)$ is the set of all ultrafilters of $B$ and $\psi(X)$ is the Boolean algebra of clopen subsets of $X$, which is also denoted by $\Delta_{1}^{0}(X)$. The usual proof of Stone duality establishes (a) and (b). Moreover $\psi \varphi(B)$ is isomorphic to $B$ and $\varphi \psi(X)$ is homeomorphic to $X$.

It remains to check that $\varphi$ and $\psi$ are Borel functions. For the Borelness of $\varphi$, identify each ultrafilter of $B$ with the underlying subset of $\mathbb{N}$, or a real in $2^{\mathbb{N}}$. For each nonempty basic open subset $\mathcal{O}$ of $2^{\mathbb{N}}, \varphi(B) \cap \mathcal{O} \neq \emptyset$ iff there is an ultrafilter of $B$ realizing the finite conditions described by $\mathcal{O}$ iff the filter generated by the finite conditions described by $\mathcal{O}$ is nontrivial, which is clearly a Borel statement. Since the Borel structure of $\mathcal{K}\left(2^{\mathbb{N}}\right)$ coincides with the Effros Borel structure, it follows that $\varphi$ is Borel.

On the other hand, given $K \in \mathcal{K}\left(2^{\mathbb{N}}\right)$, we identify $\psi(K)$ with an element of CBA in the following manner: from a canonical enumeration of the clopen subsets of $2^{\mathbb{N}},\left\{\mathcal{O}_{i}\right\}_{i \in \mathbb{N}}$, we first obtain an enumeration of the relative clopen subsets of $K$, $\left\{\mathcal{O}_{i} \cap K\right\}_{i \in \mathbb{N}}$, possibly with repetitions; letting $\rho$ be the function with domain $\mathbb{N}$ which enumerates the sequence without repetitions (but preserving the order), $\psi(K)$ can then be identified with the unique element of CBA with $\rho$ as its interpretation. The Borelness of $\psi$ then follows from the fact that the operations of union, intersection and taking complement are Borel over the space $\mathcal{K}\left(2^{\mathbb{N}}\right)$.

To establish the main theorem in section 3, we will actually prove the completeness of the homeomorphism relation between separable Boolean spaces. The topological realization of these spaces will not be closed subsets of $2^{\mathbb{N}}$; instead, they will be some zero-dimensional closed subsets of the interval $[0,1]$. Specifically, each space we construct will be the union of a countable number of points with the classical Cantor set $E_{1 / 3}$. However, this causes no problem since the above remarks about Stone duality can be modified to address these spaces. 
On several occasions we will talk about the hyperspace of compact subsets of an arbitrary compact metrizable space $X$, which we denote by $\mathcal{K}(X)$. This is defined similarly to $\mathcal{K}\left(2^{\mathbb{N}}\right)$ and for our purposes shares the same properties as $\mathcal{K}\left(2^{\mathbb{N}}\right)$.

II. The generating tree argument.

Next we give an alternative definition for the reduction function $\varphi$ above. This is another coding of countable Boolean algebras by closed subsets of $2^{\mathbb{N}}$ which will be useful in section 5. We use the concept of generating trees for countable Boolean algebras, as described in [Go97, 1.7].

First recall that in general a rooted binary tree is a partial order $\left(T, \leq^{T}\right)$ with the following properties:

(i) there is a root in $T$, i.e., some $r \in T$ such that $r \leq^{T} t$ for all $t \in T$;

(ii) for every $t \in T$ other than the root, there is a unique parent of $t$, i.e., some element in $T$, denoted by $p(t)$, such that $p(t) \leq^{T} t, t \not^{T} p(t)$ and for any $t^{\prime} \in T$ with $p(t) \leq^{T} t^{\prime} \leq^{T} t$ either $t^{\prime} \leq^{T} p(t)$ or $t \leq^{T} t^{\prime}$

(iii) for every $t \in T$, there are at most two $s \in T$ such that $p(s)=t$.

Now for a countable Boolean algebra $B$ a generating tree for $B$ is a rooted binary tree $(T, \geq)$, where $T \subseteq B$ and $\geq$ is the indicated relation on $B$, such that

(a) $1_{B} \in T$ (hence $1_{B}$ is the root of $T$ );

(b) for every $t \in T \backslash\left\{1_{B}\right\}$ there is some $t^{\prime} \neq t$ such that $p(t)=p\left(t^{\prime}\right)$;

(c) if $t, t^{\prime} \in T$ are such that $p(t)=p\left(t^{\prime}\right)$, then $t \cup t^{\prime}=p(t)$ and $t \cap t^{\prime}=0_{B}$;

(d) for any $x \in B$ there are a finite number of elements $t_{1}, \ldots, t_{n} \in T$ such that $x=t_{1} \cup \cdots \cup t_{n}$.

It follows from the definition that $0_{B}$ is not an element of any generating tree for $B$. It is also easy to check that in case $B$ contains atoms any generating tree for $B$ contains finite branches with the atoms as terminal nodes. Note also that every branch of a generating tree for $B$ corresponds in a canonical way to an ultrafilter on $B$.

The class of all rooted binary trees can be identified with the set of all subtrees of $2^{<\mathbb{N}}$ (with the empty sequence as the root). Given a subtree $T$ of $2^{<\mathbb{N}}$ such that, for any $t$ in $T$ other than the root, there is $t^{\prime} \neq t$ in $T$ with $p(t)=p\left(t^{\prime}\right)$, one can form a Boolean algebra $B$ such that (a) through (d) above are satisfied. This $B$ is said to be generated by $T$. Moreover, we can fix a Borel coding so that $B$ has universe $\mathbb{N}$ (when it is infinite, or equivalently when $T$ has an infinite branch). Conversely, it is also true that any countable Boolean algebra has a generating tree. Moreover, the proof of this fact in Go97] made it clear that there is a Borel algorithm which, given any Boolean algebra $B$ with universe $\mathbb{N}$, produces a subtree $T$ of $2^{<\mathbb{N}}$ so that the Boolean algebra generated by $T$ is isomorphic to $B$. As we remarked above, this tree $T$ might have finite branches. We extend $T$ to some subtree $T^{\prime} \supseteq T$ of $2^{<\mathbb{N}}$ so that $T^{\prime}$ does not have finite branches. This can be done in a canonical way so that if $t \in T$, then $T^{\prime}$ contains all elements $t^{\frown} 0, t^{\frown}(0,0), t^{\frown}(0,0,0)$ and so on. Finally we let $\varphi(B)$ be the set of infinite branches of $T^{\prime}$, which is a closed subset of $2^{\mathbb{N}}$. It is straightforward to check that this $\varphi$ is Borel. Furthermore, by the Stone duality we reviewed previously, an isomorphism between two Boolean algebras can be faithfully translated to a homeomorphism between their images under $\varphi$, and vice versa.

This last fact has a useful consequence. Suppose $B_{\eta}$ is a countable atomless Boolean algebra with universe $\mathbb{N}$. Then the generating tree obtained from the algorithm must be the perfect tree $2^{<\mathbb{N}}$. Moreover, the automorphisms of $B_{\eta}$ are 
in one-one correspondence with the autohomeomorphisms of the Cantor space. Let us denote these groups by $\operatorname{Aut}\left(B_{\eta}\right)$ and $\operatorname{Aut}\left(2^{\mathbb{N}}\right)$, respectively. The correspondence turns out to be an isomorphism between $\operatorname{Aut}\left(B_{\eta}\right)$ and $\operatorname{Aut}\left(2^{\mathbb{N}}\right)$ as topological groups. Thus we have obtained a proof of the folklore fact that Aut $\left(2^{\mathbb{N}}\right)$ is a closed subgroup of $S_{\infty}$.

III. The theory of Boolean algebras.

Finally let us recall some definitions and results about the first-order theories of Boolean algebras. Throughout the rest of the paper let us fix the signature for Boolean algebras to be $\langle\cup, \cap, C, 0,1\rangle$. We will also use $\backslash$ for taking relative complement, $\dot{\cup}$ for disjoint union, and $<, \leq,>, \geq$ in the natural sense. For a Boolean algebra $B$ and an element $a \in B$, we write $\hat{a}$ for the restricted subalgebra of $B$ with universe $\{b \in B \mid b \leq a\}$. We will use this notation so rarely that no mention of $B$ is really necessary. For any Boolean algebra $B$ the Fréchet ideal of $B$ is

$$
F(B)=\left\{b \in B \mid \text { there are atoms } a_{1}, \ldots, a_{n} \text { such that } b=a_{1} \cup \cdots \cup a_{n}\right\} .
$$

The most important concept we will use is the elementary characteristic for a Boolean algebra. Given a Boolean algebra B, define its Ershov-Tarski ideal

$$
I(B)=\{x \cup y \mid x \text { is atomic and } y \text { is atomless }\}
$$

(we consider $0_{B}$ here to be both atomic and atomless). Then define a sequence of Boolean algebras by induction: $B_{0}=B$ and, for $k \in \mathbb{N}, B_{k+1}=B_{k} / I\left(B_{k}\right)$. Finally define the elementary characteristic $\operatorname{ch}(B)=(p, q, r)$ of $B$ as follows:

- if for every $k \in \mathbb{N}$ the algebra $B_{k}$ is non-trivial, i.e., $B_{k} \neq\left\{0_{B_{k}}\right\}$, then define $\operatorname{ch}(B)=(\omega, 0,0)$;

- otherwise, there are some $p \in \mathbb{N}$ with $B_{p} \neq\left\{0_{B_{p}}\right\}$ but $B_{p+1}=\left\{0_{B_{p+1}}\right\}$, in which case define $\operatorname{ch}(B)=(p, q, r)$, where

$-q \leq \omega$ is the number of atoms in $B_{p}$;

$-r=1$ if $B_{p}$ contains atomless elements, and $r=0$ otherwise.

Any two Boolean algebras are elementarily equivalent iff their elementary characteristics coincide. Thus a complete theory of Boolean algebras is determined by the elementary characteristic of any of its models.

When studying the isomorphism relation for countable Boolean algebras, we will also use the concept of V-relations introduced by Vaught. Let $A$ and $B$ be Boolean algebras. A subset $R$ of $A \times B$ is a $V$-relation between $A$ and $B$ if

(i) $1_{A} R 1_{B}$

(ii) $a R 0_{B}$ implies $a=0_{A} ; 0_{A} R b$ implies $b=0_{B}$;

(iii) $a R\left(b_{1} \cup \dot{\cup} b_{2}\right)$ implies $a=a_{1} \dot{\cup} a_{2}$, where $a_{1} R b_{1}$ and $a_{2} R b_{2}$, and $\left(a_{1} \cup \dot{\cup} a_{2}\right) R b$ implies $b=b_{1} \cup \dot{\cup} b_{2}$, where $a_{1} R b_{1}$ and $a_{2} R b_{2}$.

If $A$ and $B$ are countable, then $A \cong B$ iff there is a V-relation between $A$ and $B$.

\section{The completeness of Atomic Boolean algebras}

In this section we prove the Borel completeness of the class of all countable Boolean algebras. Actually we will prove a slightly stronger result, namely that the class of countable atomic Boolean algebras (in fact, the ones with elementary characteristic $(0, \omega, 0))$ is Borel complete. This not only implies the main result we want to prove but also will be used as a basis step in the inductive proof of the next section.

For this we need some preliminary constructions. 
Throughout the rest of this section fix a surjection $a: 2 \mathbb{N} \rightarrow \mathbb{N}$ such that $a^{-1}(\{n\})$ is infinite for every $n \in \mathbb{N}$. This determines a surjection $(2 \mathbb{N})^{<\mathbb{N}} \rightarrow \mathbb{N}<\mathbb{N}$, still denoted by $a$, defined componentwise. Note that this function $a$ has the following properties:

- $a^{-1}(\{t\})$ is infinite for every $t \in \mathbb{N}<\mathbb{N} \backslash\{\emptyset\}$

- $a$ is increasing, i.e., $\forall t, u \in(2 \mathbb{N})^{<\mathbb{N}}(t \subseteq u \Rightarrow a(t) \subseteq a(u))$;

- $\forall t \in(2 \mathbb{N})^{<\mathbb{N}}$ length $(t)=$ length $(a(t))$;

- $\forall t \in(2 \mathbb{N})<\mathbb{N} \forall n \in \mathbb{N} a(t\lceil n)=a(t)\lceil n$;

- let $t \in(2 \mathbb{N})^{<\mathbb{N}}$ and assume $w \in \mathbb{N}^{<\mathbb{N}}$ is such that $a(t) \subseteq w$; then $\left\{u \in(2 \mathbb{N})^{<\mathbb{N}}\right.$ $t \subseteq u \wedge a(u)=w\}$ is infinite.

For $t \in \mathbb{N}^{<\mathbb{N}}$ let $A_{t}=a^{-1}(\{t\})$. By the remarks above we have that $A_{t} \subseteq$ $(2 \mathbb{N})^{\text {length }(t)}$ for any $t \in \mathbb{N}<\mathbb{N}$.

Let $\mathcal{I}=\left\{I_{q}\right\}_{q \in \mathbb{Q}}$ be the set of open intervals included in $[0,1]$ which are deleted to obtain the Cantor space $E_{1 / 3}$ (note indeed that $\mathcal{I}$ is totally ordered in type $\eta$ the order type of rational numbers - by the order defined as

$$
I<J \Leftrightarrow \forall x \in I \forall y \in J x<y
$$

for $I, J \in \mathcal{I})$. Fix also an enumeration $\mathbb{Q}=\left\{q_{n}\right\}_{n \in \mathbb{N}}$ of the rational numbers.

Now we define inductively a subset $\mathcal{I}_{t}=\left\{J_{t^{\frown} n}\right\}_{n \in \mathbb{N}} \subseteq \mathcal{I}$ for every $t \in \mathbb{N}<\mathbb{N}$. Let $\mathcal{I}_{\emptyset}=\left\{J_{n}\right\}_{n \in \mathbb{N}}$ be a decreasing sequence of elements of $\mathcal{I}$ such that $\lim _{n \rightarrow \infty}\left(\sup J_{n}\right)=0$. Let $h>0$ and assume that, for all $t \in \mathbb{N}^{<h}, \mathcal{I}_{t}=\left\{J_{t^{-} n}\right\}_{n \in \mathbb{N}}$ has been defined in such a way that $\forall u, v \in \mathbb{N} \leq h \quad\left(J_{u}<J_{v} \Leftrightarrow v<_{K B} u\right)$, where $<_{K B}$ is the KleeneBrouwer ordering on $\mathbb{N}<\mathbb{N}$ (see [Kec95, 2.G]). For $t \in \mathbb{N}^{h}$ we let $\mathcal{I}_{t}=\left\{J_{t^{\wedge} n}\right\}_{n \in \mathbb{N}}$ be a decreasing sequence of elements of $\mathcal{I}$ such that:

(1) $\lim _{n \rightarrow \infty}\left(\sup J_{t \frown n}\right)=\sup J_{t}$

(2) if $t$ is not constantly 0 , then $J_{t^{-0}}<J_{t^{-}}$, where $t^{-}$is the immediate predecessor of $t$ in $<_{K B} \cap(\mathbb{N} \leq h \times \mathbb{N} \leq h)$;

(3) $J_{t \frown 0}=I_{q_{l}}$ where $l$ is the smallest natural number such that $J_{t}<I_{q_{l}}$ and $I_{q_{l}}$ satisfies (2).

Note that the last condition guarantees that $\bigcup_{t \in \mathbb{N}<\mathbb{N}} \mathcal{I}_{t}=\mathcal{I}$. Denote $d_{\emptyset}=0$, and for $t \in \mathbb{N}^{<\mathbb{N}} \backslash\{\emptyset\}, d_{t}=\sup J_{t}$. Call $Q=\left\{d_{t}\right\}_{t \in \mathbb{N}<\mathbb{N}} \subseteq E_{1 / 3}$ the set of critical points and, for $t \in \mathbb{N}<\mathbb{N}$, say that the critical point $d_{t}$ has height length $(t)$.

Furthermore, for $t \in \mathbb{N}<\mathbb{N}$ say:

- $t$ is $\operatorname{good}$ if $t \in(2 \mathbb{N})^{<\mathbb{N}}$;

- $t$ is mixed if $t$ is of the form $u^{\frown}(2 n+1)$, where $u$ is good and $n \in \mathbb{N}$;

- $t$ is bad if it is not good nor mixed.

So, given $\xi \in \mathbb{N}^{\mathbb{N}}$, either $\xi\lceil m$ is good for every $m \in \mathbb{N}$, or else there is a unique $m>0$ such that $\xi\lceil m$ is mixed (and $\xi \uparrow h$ is $\operatorname{good}$ if $h<m$ and bad if $h>m$ ). In the latter case this $m$ is in fact the smallest natural number for which $\xi(m-1) \in 2 \mathbb{N}+1$. For $t \in \mathbb{N}<\mathbb{N} \backslash\{\emptyset\}$ call $J_{t}$ good, mixed or bad according to the case in which $t$ falls. Do the same for the points $d_{t}$, where $t \in \mathbb{N}<\mathbb{N}$.

Now consider the language $\mathcal{L}_{0}$ of graphs (which only consists of one binary relation symbol). For every $n \in \mathbb{N}$, let $\mathrm{TY}_{n}$ be the set of all quantifier free types for the first $n$ variables in the language $\mathcal{L}_{0} \cup\{=\}$ (such a type is a set of formulas which are atomic or negation of atomic ones, whose variables are among the first 
$n$ variables). Let $\mathrm{TY}=\bigcup_{n \in \mathbb{N}} \mathrm{TY}_{n}$. Fix an enumeration $e$ of TY such that, if $e(i) \in \mathrm{TY}_{n}$ and $e(j) \in \mathrm{TY}_{m}$, where $n<m$, then $i<j$. The type $e(i)$ is said to have been coded by $i$. For $G$ a countable graph on $\mathbb{N}$ and $t \in \mathbb{N}<\mathbb{N}$ let $\tau_{G}(t) \in \mathbb{N}$ be the code of the quantifier free type of $t$ in $G$ (or $\tau(t)$ when there is no danger of confusion). We are now ready to present our main construction.

Theorem 1. The class of countable atomic Boolean algebras is Borel complete.

Proof. We will associate to each countable graph $G$ on $\mathbb{N}$ a separable Boolean space $X_{G}$ so that $\operatorname{ch}\left(\boldsymbol{\Delta}_{1}^{0}\left(X_{G}\right)\right)=(0, \omega, 0)$ and, for $G$ and $G^{\prime}$ countable graphs, $G \cong G^{\prime} \Leftrightarrow X_{G} \cong X_{G^{\prime}}$.

Define $X_{G}$ to be the union of $E_{1 / 3}$ with countably many points, which are placed in the deleted intervals $J_{t}$ according to the following rules:

- if $t$ is good add in $J_{t}$ an increasing sequence of order type $\omega^{\tau(a(t))+2}$ converging to $d_{t}$;

- if $t$ is mixed, then there are a good sequence $u$ and an odd number $n$ such that $t=u^{\curvearrowleft} n$. Add in $J_{t}$ an increasing sequence of order type $\omega^{\tau(a(u))+2}$ converging to $d_{t}$;

- if $t$ is bad add a middle point in $J_{t}$.

For $t \in(2 \mathbb{N})^{<\mathbb{N}}$ say that the good critical point $d_{t}$ has type $\tau(a(t))$. Note indeed that, for $u \in \mathbb{N}^{<\mathbb{N}}$, every good critical point of type $\tau(u)$ in $X_{G}$ codes $\tau(u)$ in the following sense: $\tau(u)$ is the smallest $i \in \mathbb{N}$ such that, if $t \in A_{u}$, then in every relatively clopen neighbourhood $U$ of $d_{t}$ we can find relatively clopen $V \subseteq U$ with $d_{t} \notin V$ such that the Cantor-Bendixson derivative (cf. Kec95, Definition 6.10]) of $V$ is homeomorphic to the space obtained by adding to the Cantor set $E_{1 / 3}$ an increasing sequence of order type $\omega^{i+1}$ converging to 0 (its leftmost point). Moreover, being a good critical point in $X_{G}$ is also topologically characterized by this property.

Claim 1. $X_{G}$ is a separable Boolean space. $X_{G}$ is clearly separable and zerodimensional. It suffices to verify that $X_{G}$ is compact. Let $\left\{y_{n}\right\}$ be a sequence in $X_{G}$. If there are infinitely many terms in the sequence belonging to the same $J_{t}$, then there is a subsequence $\left\{y_{n_{k}}\right\}$ converging to a point added in $J_{t}$ or to $d_{t}$. If infinitely many terms are in $E_{1 / 3}$, then there is a subsequence converging in $E_{1 / 3}$. So we can assume that every term of the sequence belongs to a different $J_{t}$. By thinning we can also assume that there is a sequence $\left\{z_{n}\right\} \subseteq E_{1 / 3}$ with $\left|y_{n}-z_{n}\right|<1 / 3^{n}$, so we get a subsequence which converges to a point in $E_{1 / 3}$.

Claim 2. There is a zero-dimensional subspace $\mathcal{X}$ of $[0,1]$ such that the assignment $G \mapsto X_{G}$ takes values in $\mathcal{K}(\mathcal{X})$. Moreover, such assignment is injective and can be defined to be Borel. So the range is Borel in $\mathcal{K}(\mathcal{X})$.

Proof. The existence of $\mathcal{X}$ is given by the fact that, in the construction of $X_{G}$, for every $n \in \mathbb{N}$ there are only finitely many possible types for $n$-tuples; so, for $t \in \mathbb{N}^{n}$, there are only finitely many possible sequences that can be added in $J_{t}$ when constructing $X_{G}$. Thus, fixing a predetermined way of adding sequences in the deleted intervals, the union $\mathcal{X}$ of all the spaces $X_{G}$ turns out to be zero-dimensional.

If $G$ and $G^{\prime}$ are graphs on $\mathbb{N}$ with $G \neq G^{\prime}$, let $n, m \in \mathbb{N}$ be such that, without loss of generality, $(n, m) \in G$ while $(n, m) \notin G^{\prime}$. Then $\tau_{G}(n, m) \neq \tau_{G^{\prime}}(n, m)$ and 
thus, if $t \in(2 \mathbb{N})^{2}$ is such that $a(t)=(n, m)$, the sequence added in $J_{t}$ to obtain $X_{G}$ is different from the sequence added in the same interval for obtaining $X_{G^{\prime}}$. For the second assertion note that it is possible to check in a Borel way whether a basic clopen subset of $\mathcal{X}$ intersects $X_{G}$ using an argument as the one described in section 2 .

Claim 3. $\operatorname{ch}\left(\boldsymbol{\Delta}_{1}^{0}\left(X_{G}\right)\right)=(0, \omega, 0)$.

Proof. First notice that $\boldsymbol{\Delta}_{1}^{0}\left(X_{G}\right)$ is countably infinite, since $X_{G}$ is infinite, zerodimensional and separable. To see that it is atomic it suffices (by Ko89, Proposition 7.18]) to show that the set of isolated points in $X_{G}$ is dense. This holds since every open subset of $[0,1]$ which intersects $E_{1 / 3}$ must contain some of the deleted intervals $J_{t}$, where we have added some isolated points.

Notice now that every $x \in E_{1 / 3} \backslash Q$ determines a unique element $g(x)=\xi \in \mathbb{N}^{\mathbb{N}}$ in the sense that for every $n \in \mathbb{N}$ there is a neighbourhood $U_{n}$ of $x$ such that $\forall t \in \mathbb{N}^{<\mathbb{N}}\left(d_{t} \in U_{n} \Rightarrow \xi\lceil n \subseteq t)\right.$. More constructively, for every $n \in \mathbb{N}, d_{\xi\lceil n}$ is the critical point of height $n$ immediately at the left of $x$.

Claim 4. The function $g: E_{1 / 3} \backslash Q \rightarrow \mathbb{N}^{\mathbb{N}}$ is a homeomorphism.

Proof. Let $x, y \in E_{1 / 3} \backslash Q$ with $x<y$; this implies that there is $t \in \mathbb{N}<\mathbb{N}$ with $x<d_{t}<y$, so $g(x) \uparrow$ length $(t) \neq g(y) \uparrow$ length $(t)$, proving injectivity.

For $t \in \mathbb{N}^{<\mathbb{N}} \backslash\{\emptyset\}$ let $N_{t}=\left\{\zeta \in \mathbb{N}^{\mathbb{N}} \mid t \subseteq \zeta\right\}$ be a basic open set in $\mathbb{N}^{\mathbb{N}}$. If $t$ is not constantly zero then, letting $t^{-}$be the element of $\bigcup_{h=0}^{\operatorname{length}(t)} \mathbb{N}^{h}$ immediately preceding $t$ in $\left.<_{K B}, g^{-1}\left(N_{t}\right)=\right] d_{t}, d_{t^{-}}\left[\cap\left(E_{1 / 3} \backslash Q\right)\right.$; if $t$ is constantly zero, then $g^{-1}\left(N_{t}\right)=$ ]$d_{t}, 1\left[\cap\left(E_{1 / 3} \backslash Q\right)\right.$. In both cases $g^{-1}\left(N_{t}\right)$ is open and this proves continuity of $g$.

Now let $\xi \in \mathbb{N}^{\mathbb{N}}$ and let $x=\lim _{n \rightarrow \infty} d_{\xi\lceil n}$. Note that indeed such a sequence converges since it is bounded and increasing; moreover, $x \in E_{1 / 3} \backslash Q$ since every critical point has a left neighbourhood not containing other critical points and thus $x \notin Q$. We have $g(x)=\xi$ since, for $n \in \mathbb{N}, d_{\xi \backslash n}$ is the critical point of height $n$ immediately to the left of $x$. This gives us surjectivity.

Finally, for every $n \in \mathbb{N}$, let $\xi_{n} \in \mathbb{N}^{\mathbb{N}}$ and set $x_{n}=g^{-1}\left(\xi_{n}\right)$. Suppose $\lim _{n \rightarrow \infty} \xi_{n}=$ $\xi \in \mathbb{N}^{\mathbb{N}}$ and let $x=g^{-1}(\xi)$ with the aim of proving $\lim _{n \rightarrow \infty} x_{n}=x$. Since $g$ is continuous it is enough to check the convergence of $x_{n}$. By the hypothesis $\forall m \in$ $\mathbb{N} \exists \nu \in \mathbb{N} \forall n>\nu \xi\left\lceil m \subseteq \xi_{n}\right.$ and this implies $d_{\xi\lceil m}<x_{n}<z_{m}$, for all $n>\nu$, where $z_{m}=d_{(\xi\lceil m)}$ - if $\xi \uparrow m$ is not constantly zero and $z_{m}=1$ otherwise. Since $\lim _{m \rightarrow \infty}\left(d_{\xi\lceil m}-z_{m}\right)=0$ (because $d_{\xi\lceil m}$ is increasing, $z_{m}$ is nonincreasing and every critical point is indexed by a finite sequence), we get that $x_{n}$ converges.

We can extend the function $g$ to a bijection $g: E_{1 / 3} \rightarrow \mathbb{N}<\mathbb{N} \cup \mathbb{N}^{\mathbb{N}}=\mathbb{N}<\omega+1$ defining $\forall t \in \mathbb{N}^{<\mathbb{N}} g\left(d_{t}\right)=t$ and via $g$ we can endow $\mathbb{N}<\mathbb{N} \cup \mathbb{N}^{\mathbb{N}}=\mathbb{N}^{<\omega+1}$ with a Polish topology homeomorphic to the Cantor space in which $\mathbb{N}^{\mathbb{N}}$ is a dense $G_{\delta}$ subspace (see also [Kec95 Exercise 4.16]).

Claim 5. Let $G$ and $G^{\prime}$ be graphs on $\mathbb{N}$. Then $G \cong G^{\prime} \Rightarrow X_{G} \cong X_{G^{\prime}}$. 
Proof. Let $\psi: G \rightarrow G^{\prime}$ be an isomorphism, so that $\forall t \in \mathbb{N}<\mathbb{N} \tau_{G}(t)=\tau_{G^{\prime}}(\psi(t))$. Let $\theta: \mathbb{N}<\mathbb{N} \rightarrow \mathbb{N}<\mathbb{N}$ be the bijection defined as follows: for every $u \in \mathbb{N}<\mathbb{N}$ notice that both $a^{-1}(\{u\})$ and $a^{-1}(\{\psi(u)\})$ with the lexicographic order have order type $\omega^{\text {length }(u)}$, so let $\theta$ match their points in that order; since $(2 \mathbb{N})^{<\mathbb{N}}=$ $\bigcup_{u \in \mathbb{N}<\mathbb{N}} a^{-1}(\{u\})=\bigcup_{u \in \mathbb{N}<\mathbb{N}} a^{-1}(\{\psi(u)\})$, these unions being disjoint, will take care of the good sequences. If $t \in \mathbb{N}<\mathbb{N}$ is not good, then there are $v, w \in \mathbb{N}<\mathbb{N}$ with $v$ good, $t=v^{\frown} w$ and $w(0) \in 2 \mathbb{N}+1$; define in this case $\theta(t)=\theta(v)^{\frown} w$. So $\theta$ and $\theta^{-1}$ turn out to be increasing bijections $\mathbb{N}<\mathbb{N} \rightarrow \mathbb{N}<\mathbb{N}$ with $\forall t \in \mathbb{N}<\mathbb{N}$ length $(\theta(t))=\operatorname{length}(t)$ which match good, mixed and bad sequences with good, mixed and bad sequences respectively; by the above, $\theta$ can be extended to a homeomorphism $\Theta: \mathbb{N}^{<\omega+1} \rightarrow$ $\mathbb{N}^{<\omega+1}$ that can be viewed as a homeomorphism $\Theta: E_{1 / 3} \rightarrow E_{1 / 3}$ which, in particular, for $t \in \mathbb{N}<\mathbb{N}$, sends $d_{t}$ to $d_{\theta(t)}$. Now notice that $\forall t \in(2 \mathbb{N})^{<\mathbb{N}} \tau_{G}(a(t))=$ $\tau_{G^{\prime}}(a \theta(t))$ (the former is the type in $G$ of the critical point $d_{t}$, the latter is the type in $G^{\prime}$ of $\left.d_{\theta(t)}\right)$; that is, $\Theta$ preserves the types of the good critical points. The reason is that $a \theta(t)=\psi a(t)$. So we can extend $\Theta$ to a function $\Psi: X_{G} \rightarrow X_{G^{\prime}}$ as follows. Send, for $t \in(2 \mathbb{N})^{<\mathbb{N}} \backslash\{\emptyset\}$, the sequence of order type $\omega^{\tau_{G}(a(t))+2}$ sitting in $J_{t} \cap X_{G}$ to the sequence (of the same order type) sitting in $J_{\theta(t)} \cap X_{G^{\prime}}$. If $t \in \mathbb{N}<\mathbb{N}$ is a mixed sequence, then $t=u^{\curvearrowleft} n$ for some good sequence $u$ and odd number $n$; send the sequence of order type $\omega^{\tau(a(u))+2}$ sitting in $J_{t}$ to the sequence sitting in $J_{\theta(t)}$ of the same order type. Finally, if $t \in \mathbb{N}<\mathbb{N}$ is bad, let $\Psi$ send the point sitting in the middle of $J_{t}$ to the point sitting in the middle of $J_{\theta(t)} . \Psi$ is the required homeomorphism.

Claim 6. Let $G$ and $G^{\prime}$ be graphs on $\mathbb{N}$. Then $X_{G} \cong X_{G^{\prime}} \Rightarrow G \cong G^{\prime}$.

Proof. Assume $\Psi: X_{G} \rightarrow X_{G^{\prime}}$ is a homeomorphism. This implies that every good critical point in $X_{G}$ is sent by $\Psi$ to a good critical point in $X_{G^{\prime}}$ and the type in $G$ of the former is equal to the type in $G^{\prime}$ of the latter. We want to define a bijection $\psi: \mathbb{N} \rightarrow \mathbb{N}$ such that $\tau_{G}(t)=\tau_{G^{\prime}}(\psi(t))$ for all $t \in \mathbb{N}^{<\mathbb{N}}$; for this we shall use a back and forth argument.

Consider first $m_{0}=0=\min \mathbb{N}$ and $k_{0} \in A_{m_{0}}$; take the good critical point $p_{0}=d_{k_{0}}$ in $X_{G}$, which has type $\tau_{G}\left(m_{0}\right)$, and let $p_{0}^{\prime}=\Psi\left(p_{0}\right)$. Then $p_{0}^{\prime}$ is a good critical point and the type of $p_{0}^{\prime}$ in $G^{\prime}$ is also $\tau_{G}\left(m_{0}\right)$. Now $\exists k_{0}^{\prime} \in 2 \mathbb{N} p_{0}^{\prime}=d_{k_{0}^{\prime}}$ and so, letting $m_{0}^{\prime}=a\left(k_{0}^{\prime}\right)$, we have $\tau_{G}\left(m_{0}\right)=\tau_{G^{\prime}}\left(m_{0}^{\prime}\right)$.

Now let $m_{1}^{\prime}=\min \left(\mathbb{N} \backslash\left\{m_{0}^{\prime}\right\}\right)$ and consider $\left(m_{0}^{\prime}, m_{1}^{\prime}\right) \in \mathbb{N}^{2} \subseteq \mathbb{N}<\mathbb{N}$. Let $V_{0}$ be the intersection of $X_{G^{\prime}}$ with an open interval of ]0,1[ containing $p_{0}^{\prime}$ and small enough so that it does not intersect any $J_{r}$, for $r \in \mathbb{N}$ and $r \neq k_{0}^{\prime}$, and such that every critical point in $V_{0}$ of height 2 is sent by $\Psi^{-1}$ to a critical point of height 2 of the sequence of critical points in $X_{G}$ converging to $p_{0}$, i.e. the sequence $\left\{d_{\left(k_{0}, n\right)}\right\}$. Let $k_{1}^{\prime} \in A_{m_{1}^{\prime}}$ be such that $p_{1}^{\prime}={ }_{\text {def }} d_{\left(k_{0}^{\prime}, k_{1}^{\prime}\right)} \in V_{0}$ and set $p_{1}=\Psi^{-1}\left(p_{1}^{\prime}\right)$. Let $k_{1} \in 2 \mathbb{N}$ be such that $p_{1}=d_{\left(k_{0}, k_{1}\right)}$ and put $m_{1}=a\left(k_{1}\right)$. The same argument as in the preceding paragraph shows that $\tau_{G}\left(m_{0}, m_{1}\right)=\tau_{G^{\prime}}\left(m_{0}^{\prime}, m_{1}^{\prime}\right)$. In particular, we must have $m_{0} \neq m_{1}$.

Next let $m_{2}=\min \left(\mathbb{N} \backslash\left\{m_{0}, m_{1}\right\}\right)$ and let $V_{1}$ be the intersection of $X_{G}$ with an open interval around $p_{1}$ small enough so that it does not intersect any $J_{r}$, for $r \in \mathbb{N}^{2}$ except $J_{\left(k_{0}, k_{1}\right)}$ and such that every critical point in $V_{1}$ of height 3 is sent by $\Psi$ to a critical point of height 3 of the sequence in $X_{G^{\prime}}$ converging to $p_{1}^{\prime}$, i.e. the sequence $\left\{d_{\left(k_{0}^{\prime}, k_{1}^{\prime}, n\right)}\right\}$. Let $k_{2} \in 2 \mathbb{N}$ be such that $\left(k_{0}, k_{1}, k_{2}\right) \in A_{\left(m_{0}, m_{1}, m_{2}\right)}$ and 
$p_{2}={ }_{\text {def }} d_{\left(k_{0}, k_{1}, k_{2}\right)} \in V_{1}$ and set $p_{2}^{\prime}=\Psi\left(p_{2}\right)$. Let $k_{2}^{\prime} \in 2 \mathbb{N}$ be such that $p_{2}^{\prime}=d_{\left(k_{0}^{\prime}, k_{1}^{\prime}, k_{2}^{\prime}\right)}$ and put $m_{2}^{\prime}=a\left(k_{2}^{\prime}\right)$. Then $\tau_{G}\left(m_{0}, m_{1}, m_{2}\right)=\tau_{G^{\prime}}\left(m_{0}^{\prime}, m_{1}^{\prime}, m_{2}^{\prime}\right)$ and $m_{0}^{\prime} \neq m_{2}^{\prime} \neq m_{1}^{\prime}$.

Proceed this way, back and forth.

Define $\psi\left(m_{n}\right)=m_{n}^{\prime}$ for all $n$. To show that $\psi$ is an isomorphism between $G$ and $G^{\prime}$ observe that, for $i, j \in \mathbb{N},(i, j)$ and $(\psi(i), \psi(j))$ satisfy the same atomic and negation of atomic formulas in $G$ and $G^{\prime}$ respectively, so $(i, j) \in G$ iff $(\psi(i), \psi(j)) \in$ $G^{\prime}$; moreover, the back and forth argument guarantees that $\psi$ is a bijection.

In particular, Theorem 1 implies the announced main results:

Theorem. The class of all countable Boolean algebras is Borel complete

and

Theorem. The homeomorphism relation for separable Boolean spaces is Borel complete.

Let us remark that the construction given above was aimed to obtain separable Boolean spaces $X_{G}$ where the isolated points form a dense subset, in order to get the Stone spaces of atomic Boolean algebras (see Claim 3 above). If we are just interested in the result of the main theorem we can simplify the construction as follows: for $t \in(2 \mathbb{N})^{<\mathbb{N}}$ and $n \in 2 \mathbb{N}+1$ add in $J_{t}$ (if $t \neq \emptyset$ ) and in $J_{t^{-} n}$ an increasing sequence of order type $\omega^{\tau(a(t))+1}$ converging to $d_{t}$ and $d_{t^{-} n}$ respectively; if $t \in \mathbb{N}<\mathbb{N}$ is a bad sequence do not add anything in $J_{t}$. Now, for $u \in \mathbb{N}<\mathbb{N}$, a good critical point $d \in X_{G}$ of type $\tau(u)$ is topologically characterised by the fact that $\tau(u)$ is the smallest $i \in \mathbb{N}$ such that each clopen neighbourhood $U$ of $d$ contains some clopen subset $V$ with $d \notin V$ and $V$ homeomorphic to the space obtained by adding to the Cantor space $E_{1 / 3}$ an increasing sequence of order type $\omega^{i+1}$ converging to 0 .

Given the abundance of Borel complete classes, one could seek to simplify the proof of the main theorem by trying to find a more direct reduction of some Borel complete class to that of Boolean algebras. For example, a reduction from linear orderings to Boolean algebras would assign to each countable linear ordering $L$ some countable Boolean algebra $B(L)$ so that

$$
L \cong L^{\prime} \Leftrightarrow B(L) \cong B\left(L^{\prime}\right) .
$$

Boolean algebraists are quite familiar with a candidate of such an assignment, i.e., the interval algebras. However, Hanf ([Ha76] $)$ showed that in a very strong sense, this assignment fails to be a reduction.

There is, nevertheless, some connection between an open problem in [FS89] and a possible strategy for an alternative proof of Theorem 1. In [FS89 the authors considered an increasing (in the sense of Borel reducibility) $\omega_{1}$-sequence of Borel equivalence relations, denoted by $\left\{\mathcal{I}_{\alpha}\right\}_{\alpha \in \omega_{1}}$, where, for $\alpha \in \omega_{1}, \mathcal{I}_{\alpha}$ is the class of well-founded trees of rank $\leq \alpha$. They asked whether, for a class $A$ of countable structures, $\mathcal{I}_{\alpha} \leq_{B} A$ for all $\alpha<\omega_{1}$ implies that $A$ is Borel complete.

We observe that, in our terminology, [Ha76] actually showed $\mathcal{I}_{4} \leq_{B}$ CBA. We can sketch his proof as follows.

First notice that, as remarked by [FS89], $\mathcal{I}_{2}$ can be viewed as $\mathbb{N}, \mathcal{I}_{3}$ as the set of reals (countable subsets of $\mathbb{N}$ ), $\mathcal{I}_{4}$ as the set of countable subsets of reals and so on.

Now, for each $n \in \mathbb{N}$ consider a separable Boolean space $C_{n}$ such that, if $n$ and $m$ are different natural numbers, then $C_{n}$ and $C_{m}$ are not homeomorphic. This reduces equality in $\mathbb{N}$ (that is isomorphism in $\mathcal{I}_{2}$ ) to isomorphism in CBA. 
For each real number $x(\emptyset \neq x \subseteq \mathbb{N})$ let $D_{x}$ be the one point compactification of the disjoint union of countably many copies of each of the spaces $C_{n}$, for $n \in x$. This reduces equality in $\mathbb{R}$ (isomorphism in $\mathcal{I}_{3}$ ) to isomorphism in CBA.

Now, if $\xi$ is a countable set of reals, let $B(\xi)$ be the one point compactification of the disjoint union of countably many copies of each of the spaces $D_{x}$, for $x \in \xi$. This shows $\mathcal{I}_{4} \leq_{B}$ CBA.

Hanf's argument stops here, but the construction can be carried on. Note that indeed the same argument works also at limit steps $\lambda$ : just take one point compactifications of countably many copies of each one of the spaces corresponding to the elements of the object $x \in \mathcal{I}_{\lambda}$ under consideration, these spaces having been already constructed at previous steps.

Thus if the question of Friedman and Stanley has a positive answer, we get another proof of the Borel completeness of countable Boolean algebras. In addition, if we define our initial spaces $C_{n}$, for $n \in \mathbb{N}$, to have a dense subset of isolated points (adding for instance an increasing sequence of order type $\omega^{n+1}$ for each critical point in $E_{1 / 3}$, letting the sequence converge to it) we would get a simpler proof of our Theorem 1 .

\section{A Very STRONG FORM of the VAught COnjecture}

This section is devoted to the proof of the following theorem.

Theorem 2. Let $T$ be a complete consistent first-order theory of Boolean algebras. Then either $T$ has only one countable model up to isomorphism, or else $\operatorname{Mod}(T)$ is Borel complete.

First recall that any complete theory of Boolean algebras is completely determined by the elementary characteristic of any of its models. Our strategy for a proof of Theorem 2 is to examine every possible elementary characteristic and show that the complete theory of Boolean algebras associated to it either has only one model of cardinality less than or equal to $\aleph_{0}$, up to isomorphism, or has completely many of them. We will use extensively the result of Theorem 1, which we restate now by saying that there is a Borel reduction $\Phi: G \mapsto B_{G}$ assigning to each countable graph $G$ a countable atomic Boolean algebra $B_{G}$ in such a way that, for $G$ and $G^{\prime}$ countable graphs, $G \cong G^{\prime} \Leftrightarrow B_{G} \cong B_{G^{\prime}}$.

We need also some other preliminaries. For a linear order $L$ and $a, b \in L$ with $a<b$, we denote by $\left[a, b\left[^{L}\right.\right.$ the interval $\{x \in L \mid a \leq x<b\}$. If the linear order $L$ is clear from the context, we shall simply write $\left[a, b\left[\right.\right.$ for $\left[a, b\left[^{L}\right.\right.$. If $L_{1}$ and $L_{2}$ are two linear orders, We define the sum $L_{1}+L_{2}$ to be the linear order with universe $L_{1} \dot{\cup} L_{2}$ such that the elements of $L_{1}$ precede those of $L_{2}$. In a similar manner we can define sums of infinitely many linear orders, in symbols $\sum_{h=1}^{\infty} L_{h}$, in which the elements in $L_{h}$ precede those in $L_{h+1}$. The product $L_{1} L_{2}$ is the linear order with universe $L_{1} \times L_{2}$ and the anti-lexicographic order. By induction we can thus define the power $L^{n}$ of a linear order $L$.

Any linear order of the form $1+L+1$ (i.e., with both a smallest and a largest element) generates an interval algebra, that is the Boolean algebra whose elements are finite unions of intervals of the form $[a, b[\subseteq 1+L+1$. Following [Iv91], this algebra is denoted by $D(1+L+1)$. It is well known (cf. [Ko89, Corollary 15.10]) that any countable Boolean algebra is isomorphic to an interval algebra. Moreover, for 
a countable Boolean algebra $B$ with universe $\mathbb{N}$ we can pick in a Borel way a linear order $L_{B}$ (with universe $\mathbb{N}$ ) such that $B \cong D\left(1+L_{B}+1\right)$ (cf. Go97. Theorem 1.6.1]). Composing this with the assignment $G \mapsto B_{G}$ we get a Borel function $G \mapsto L(G)=L_{B_{G}}$ from countable graphs to countable linear orders. It follows from Theorem 1 that for countable graphs $G$ and $G^{\prime}, G \cong G^{\prime}$ iff $D(1+L(G)+1) \cong$ $D\left(1+L\left(G^{\prime}\right)+1\right)$.

For a linear order of the form $1+L+1$ we shall denote by $\pm \infty$ the largest and the smallest element, respectively (and make sure the context always clarifies what $L$ is). By convention, $D(1+\eta+1)=B_{\eta}$ is the only countable atomless Boolean algebra up to isomorphism. We also let $L_{0}=(1+\eta+1) \zeta$, where $\zeta=\omega^{*}+\omega$ is the order type of the integers.

Proposition 1. The complete theory of Boolean algebras with elementary characteristic $(0, \omega, 0)$ has completely many countable models.

Proof. This is exactly the statement of Theorem 1.

Proposition 2. The complete theory of Boolean algebras with elementary characteristic $(0, \omega, 1)$ has completely many countable models.

Proof. Consider the Borel function $G \mapsto B_{G} \times B_{\eta}$ from countable graphs to countable Boolean algebras and note that $\operatorname{ch}\left(B_{G} \times B_{\eta}\right)=(0, \omega, 1)$.

Let $G$ and $G^{\prime}$ be countable graphs. If $G \cong G^{\prime}$, then $B_{G} \cong B_{G^{\prime}}$ by Theorem 1 , and therefore $B_{G} \times B_{\eta} \cong B_{G^{\prime}} \times B_{\eta}$. Assume conversely that $\psi$ is an isomorphism between $B_{G} \times B_{\eta}$ and $B_{G^{\prime}} \times B_{\eta}$. Note that an element $(a, b) \in B_{G} \times B_{\eta}$ is atomic if and only if $b=0_{B_{\eta}}$ and the same holds for elements in $B_{G^{\prime}} \times B_{\eta}$. Since $\psi$ matches atomic elements with atomic elements, we conclude that $\psi$ determines an isomorphism from $B_{G}$ onto $B_{G^{\prime}}$.

Proposition 3. For $m>0$ the complete theory of Boolean algebras with elementary characteristic $(1, m, 0)$ has completely many countable models.

Proof. We introduce the following terminology. Let $B$ be an atomic Boolean algebra and let $a, a^{\prime} \in B$. Call $a$ finite if it is the disjunction of finitely many atoms, that is, $a \in F(B)$ where $F(B)$ is the Fréchet ideal of $B$; say $a$ is infinite if $a \notin F(B)$. Say that $a$ is cofinite in $a^{\prime}$ if $a \leq a^{\prime}$ and $a^{\prime} \backslash a$ is finite and $a$ is cofinite if it is cofinite in $1_{B}$. Notice that if $B=D(1+L+1)$, then $a \in B$ is finite (or cofinite) iff $a$ is finite (or cofinite) as a subset of the linear order.

Fix $m>0$. For each countable graph $G$, let $\Lambda_{m}(G)=L(G)+(\omega+\eta) m$ and $A_{m}(G)=D\left(1+\Lambda_{m}(G)+1\right)$. It is easy to see that $A_{m}(G) \cong B_{G} \times(D(1+\omega+\eta+1))^{m}$. Since $\operatorname{ch}(D(1+\omega+\eta+1))=(1,1,0)$, it follows that $\operatorname{ch}\left(A_{m}(G)\right)=(1, m, 0)$.

If $G \cong G^{\prime}$, then $B_{G} \cong B_{G^{\prime}}$ and therefore $A_{m}(G) \cong A_{m}\left(G^{\prime}\right)$.

Conversely, assume that $\Phi$ is the graph of an isomorphism $\varphi$ between $A_{m}(G)$ and $A_{m}\left(G^{\prime}\right)$. We define a V-relation $R \subseteq D(1+L(G)+1) \times D\left(1+L\left(G^{\prime}\right)+1\right)$.

Note that $1+L(G)+1$ is an initial segment of $1+\Lambda_{m}(G)+1$. To avoid confusion, we let $-\infty$ and $+\infty$ be the first and last element of the latter total order and let $O$ be the last element of the former order.

We now define $R$. For $A \in D(1+L(G)+1)$ and $B \in D\left(1+L\left(G^{\prime}\right)+1\right)$, set $A R B$ if one of the following holds:

- $\operatorname{card}(A)=\operatorname{card}(B) \in \mathbb{N} ;$ or

- $A$ and $B$ are infinite and there are $A^{\prime} \in D(1+L(G)+1)$ cofinite in $A$ and $B^{\prime} \in D\left(1+L\left(G^{\prime}\right)+1\right)$ cofinite in $B$ such that $A^{\prime} \Phi B^{\prime}$. 
First we verify that $[-\infty, O[R[-\infty, O[$. For this observe that $\varphi([-\infty, O[)$ must be an atomic element, so it is a finite disjoint union in $1+\Lambda_{m}\left(G^{\prime}\right)+1$ which does not contain any subinterval of order type $\eta$. This implies that $\varphi([-\infty, O[) \backslash[-\infty, O[$ is finite. Similarly, $\varphi^{-1}([-\infty, O[) \backslash[-\infty, O$ [ is finite as well. This means that there is a cofinite element in $[-\infty, O[$ mapped by $\varphi$ to a cofinite element in $[-\infty, O[$, so $[-\infty, O[R[-\infty, O[$.

It is easy to see that $A R \emptyset \Rightarrow A=\emptyset$ and $\emptyset R B \Rightarrow B=\emptyset$.

Now assume $A R B$ and $B=B_{1} \cup B_{2}$. Consider various cases. If $A$ and $B$ are finite and have the same cardinality, split $A$ into two disjoint pieces $A_{1}$ and $A_{2}$ matching the cardinalities of $B_{1}$ and $B_{2}$, respectively. Next assume that $A$ and $B$ are infinite. So there are cofinite $A^{\prime} \subseteq A$ and $B^{\prime} \subseteq B$ with $A^{\prime} \Phi B^{\prime}$. If $B_{1}$ is finite, let $A_{1}$ be a union of $\operatorname{card}\left(B_{1}\right)$ many atoms and $A_{2}=A \backslash A_{1}$; then setting $U=\left(A_{2} \cap A^{\prime}\right) \backslash \varphi^{-1}\left(B_{1}\right)$ and $V=\varphi(U)=\left(B_{2} \cap B^{\prime}\right) \backslash \varphi\left(A_{1}\right)$ we have that $U$ and $V$ are cofinite in $A_{2}$ and $B_{2}$ respectively and $U \Phi V$. A similar argument works in the case when $B_{2}$ is finite. If both $B_{1}$ and $B_{2}$ are infinite, let $B_{1}^{\prime}=B^{\prime} \cap B_{1}$ and $B_{2}^{\prime}=B^{\prime} \cap B_{2}$. Note that these are cofinite in $B_{1}$ and $B_{2}$ respectively. Let $A_{1}^{\prime}=\varphi^{-1}\left(B_{1}^{\prime}\right)$ and $A_{2}^{\prime}=\varphi^{-1}\left(B_{2}^{\prime}\right)$. Then we have $A_{1}^{\prime} \dot{\cup} A_{2}^{\prime}=A^{\prime}$. Let $A_{1}$ and $A_{2}$ be such that $A_{1}^{\prime} \subseteq A_{1}, A_{2}^{\prime} \subseteq A_{2}$ and $A_{1} \cup A_{2}=A$ (for instance $A_{1}=A_{1}^{\prime}$ and $\left.A_{2}=A \backslash A_{1}^{\prime}\right)$. We then have $A_{1} R B_{1}$ and $A_{2} R B_{2}$.

By symmetry, $R$ is a $V$-relation. This gives us the existence of an isomorphism between $D(1+L(G)+1)$ and $D\left(1+L\left(G^{\prime}\right)+1\right)$, and therefore $B_{G} \cong B_{G^{\prime}}$. By Theorem 1 we have $G \cong G^{\prime}$.

Proposition 4. For $m \in \mathbb{N}$ the complete theory of Boolean algebras with elementary characteristic $(1, m, 1)$ has completely many countable models.

Proof. For each countable graph $G$ we associate the interval algebra $D\left(1+\Lambda_{m}(G)+\right.$ $\left.1+L_{0} \eta+1\right)$, which is isomorphic to $B_{G} \times(D(1+\omega+\eta+1))^{m} \times D\left(1+L_{0} \eta+1\right)$ and has elementary characteristic $(1, m, 1)$. For the rest of the proof the argument is the same as in the proof of Proposition 3.

Proposition 5. Let $p<\omega, q \leq \omega$ and $r \in\{0,1\}$. Assume that the complete theory of Boolean algebras with elementary characteristic $(p, q, r)$ has completely many countable models. Then this holds for the complete theory of Boolean algebras with elementary characteristic $(p+1, q, r)$ as well.

Proof. Let $G \mapsto A_{G}$ be a Borel reduction from countable graphs to countable Boolean algebras with elementary characteristic $(p, q, r)$. We define a reduction $G \mapsto A_{G}^{*}$ whose values have elementary characteristic $(p+1, q, r)$. For $G$ a countable graph let $\lambda(G)=L_{A_{G}}$ and $A_{G}^{*}=D\left(1+L_{0} \lambda(G)+1\right)$. We verify that this works.

First, by [Iv91], if $L$ is a countable linear order such that $\operatorname{ch}(D(1+L+1))=$ $(p, q, r)$, then $\operatorname{ch}\left(D\left(1+L_{0} L+1\right)\right)=(p+1, q, r)$. It is also verified in [Iv91] that, for $L$ and $L^{\prime}$ countable total orders, $D(1+L+1) \varsubsetneqq D\left(1+L^{\prime}+1\right) \Rightarrow D\left(1+L_{0} L+1\right) \varsubsetneqq$ $D\left(1+L_{0} L^{\prime}+1\right)$.

For our purpose it suffices to show the converse, namely $D(1+L+1) \cong$ $D\left(1+L^{\prime}+1\right) \Rightarrow D\left(1+L_{0} L+1\right) \cong D\left(1+L_{0} L^{\prime}+1\right)$.

So let $\Phi$ be the graph of an isomorphism from $D(1+L+1)$ onto $D\left(1+L^{\prime}+1\right)$. Again we define a $V$-relation $R \subseteq D\left(1+L_{0} L+1\right) \times D\left(1+L_{0} L^{\prime}+1\right)$. 
Let $A \in D\left(1+L_{0} L+1\right)$ and $B \in D\left(1+L_{0} L^{\prime}+1\right)$. $A$ and $B$ can be decomposed as disjont unions of intervals

$$
A=\bigcup_{\alpha=1}^{h} I_{\alpha} \cup \bigcup_{\beta=1}^{k} J_{\beta} \cup \bigcup_{\gamma=1}^{l} K_{\gamma} \text { and } B=\bigcup_{\alpha^{\prime}=1}^{h^{\prime}} I_{\alpha^{\prime}}^{\prime} \cup \bigcup_{\beta^{\prime}=1}^{k^{\prime}} J_{\beta^{\prime}}^{\prime} \cup \bigcup_{\gamma^{\prime}=1}^{l^{\prime}} K_{\gamma^{\prime}}^{\prime}
$$

where

- $I_{\alpha}$ is of the form $[(x, n, a),(y, m, b)[$ with $a<b,[-\infty,(y, m, b)[$ or $[(x, n, a)$, $+\infty[$ (we will call this an interval of the first type);

- $J_{\beta}$ is of the form $[(x, n, a),(y, n, a)$ [ (we will call this an interval of the second type);

- $K_{\gamma}$ is of the form $[(+\infty, n, a),(-\infty, n+1, a)$ [ (we will call this an interval of the third type)

and similarly for $I_{\alpha^{\prime}}^{\prime}, J_{\beta^{\prime}}^{\prime}, K_{\gamma^{\prime}}^{\prime}$. Such a decomposition is not unique, but we have the following:

- $h$ being 0 is independent of the decomposition, since it says whether $A$ contains finitely many or infinitely many atoms;

- define a mapping $*: D\left(1+L_{0} L+1\right) \rightarrow D(1+L+1)$ by letting $[(x, n, a)$, $(y, m, b)\left[^{*}=\left[a, b\left[\right.\right.\right.$ for $a<b,\left[-\infty,(y, m, b)\left[^{*}=\left[-\infty, b\left[\right.\right.\right.\right.$ and $\left[(x, n, a),+\infty\left[^{*}=\right.\right.$ $\left[a,+\infty\left[\right.\right.$, then $A^{*}=\bigcup_{\alpha=1}^{h} I_{\alpha}^{*} \subseteq 1+L+1$ is independent of the decomposition, since an interval of the first type is not a finite union of intervals of the second and third types;

- if $h=0$, then $k$ being 0 is independent of the decomposition, since in this case this tells whether $A$ contains atomless elements;

- if $h=0$, then $l$ is independent of the decomposition, since in this case $l$ is the number of atoms contained in $A$.

Now define $A R B$ if and only if the following conditions hold:

- $\bigcup_{\alpha=1}^{h} I_{\alpha}^{*} \Phi \bigcup_{\alpha^{\prime}=1}^{h^{\prime}}\left(I_{\alpha^{\prime}}^{\prime}\right)^{*}$ (this implies in particular $\left.h=0 \Leftrightarrow h^{\prime}=0\right)$;

- if $h=h^{\prime}=0$, then $\left(k=0 \Leftrightarrow k^{\prime}=0\right)$ and $l=l^{\prime}$.

We prove now that $R$ works. Assume $A R B \dot{\cup} C, A$ and $B$ are as before and similarly,

$$
C=\bigcup_{\alpha^{\prime \prime}=1}^{h^{\prime \prime}} I_{\alpha^{\prime \prime}}^{\prime \prime} \cup \bigcup_{\beta^{\prime \prime}=1}^{k^{\prime \prime}} J_{\beta^{\prime \prime}}^{\prime \prime} \cup \bigcup_{\gamma^{\prime \prime}=1}^{l^{\prime \prime}} K_{\gamma^{\prime \prime}}^{\prime \prime}
$$

We want to find $A^{\prime}$ and $A^{\prime \prime}$ in $D\left(1+L_{0} L+1\right)$ such that $A^{\prime} \dot{\cup} A^{\prime \prime}=A$ and $A^{\prime} R B$ and $A^{\prime \prime} R C$.

Assume $h=h^{\prime}=h^{\prime \prime}=0$, so that $l=l^{\prime}+l^{\prime \prime}$. If $k=k^{\prime}=k^{\prime \prime}=0$ let

$$
A^{\prime}=\bigcup_{\gamma=1}^{l^{\prime}} K_{\gamma} \text { and } A^{\prime \prime}=\bigcup_{\gamma=l^{\prime}+1}^{l} K_{\gamma} \text {. }
$$

If $k^{\prime} \neq 0=k^{\prime \prime}$ let

$$
A^{\prime}=\bigcup_{\beta=1}^{k} J_{\beta} \cup \bigcup_{\gamma=1}^{l^{\prime}} K_{\gamma} \text { and } A^{\prime \prime}=\bigcup_{\gamma=l^{\prime}+1}^{l} K_{\gamma}
$$


and similarly if $k^{\prime}=0 \neq k^{\prime \prime}$. If $k^{\prime} \neq 0 \neq k^{\prime \prime}$, split $J_{1}$ in two disjoint subintervals $J$ and $\hat{J}$ and let

$$
A^{\prime}=J \cup \bigcup_{\gamma=1}^{l^{\prime}} K_{\gamma} \text { and } A^{\prime \prime}=\hat{J} \cup \bigcup_{\beta=2}^{k} J_{\beta} \cup \bigcup_{\gamma=l^{\prime}+1}^{l} K_{\gamma} .
$$

Assume now $h \neq 0$. We have

$$
\bigcup_{\alpha=1}^{h} I_{h}^{*} \Phi \bigcup_{\alpha^{\prime}=1}^{h^{\prime}}\left(I_{\alpha^{\prime}}^{\prime}\right)^{*} \cup \bigcup_{\alpha^{\prime \prime}=1}^{h^{\prime \prime}}\left(I_{\alpha^{\prime \prime}}^{\prime \prime}\right)^{*}
$$

If $h^{\prime}=0$, let $P_{1}, \ldots, P_{k^{\prime}}$ be disjoint intervals of the second type included in $I_{1}$ and let $Q_{1}, \ldots, Q_{l^{\prime}}$ be disjoint intervals of the third type included in $I_{1}$. Let

$$
I=I_{1} \backslash\left(\bigcup_{i=1}^{k^{\prime}} P_{i} \cup \bigcup_{j=1}^{l^{\prime}} Q_{j}\right)=\bigcup_{\hat{\alpha}=1}^{\hat{h}} X_{\hat{\alpha}} \cup \bigcup_{\hat{\beta}=1}^{\hat{k}} Y_{\hat{\beta}} \cup \bigcup_{\hat{\gamma}=1}^{\hat{l}} Z_{\hat{\gamma}}
$$

where $X_{\hat{\alpha}}, Y_{\hat{\beta}}, Z_{\hat{\gamma}}$ are intervals of the first, second and third types respectively.

Note that $\bigcup_{\hat{\alpha}=1}^{\hat{h}} X_{\hat{\alpha}}^{*}=I_{1}^{*}$. Put $A^{\prime}=\bigcup_{i=1}^{k^{\prime}} P_{i} \cup \bigcup_{j=1}^{l^{\prime}} Q_{j}$ and $A^{\prime \prime}=A \backslash A^{\prime}$. Similarly, if $h^{\prime \prime}=0$.

Now let $h^{\prime} \neq 0 \neq h^{\prime \prime}$. There are positive natural numbers $u$ and $v$ and disjoint nonempty intervals $U_{1}, \ldots, U_{u}, V_{1}, \ldots V_{v}$, every one being included in one of the intervals $I_{\alpha}^{*}$, such that $\bigcup_{\xi=1}^{u} U_{\xi} \cup \bigcup_{\rho=1}^{v} V_{\rho}=\bigcup_{\alpha=1}^{h} I_{h}^{*}$ and $\bigcup_{\xi=1}^{u} U_{\xi} \Phi \bigcup_{\alpha^{\prime}=1}^{h^{\prime}}\left(I_{\alpha^{\prime}}^{\prime}\right)^{*}$ and $\bigcup_{\rho=1}^{v} V_{\rho} \Phi \bigcup_{\alpha^{\prime \prime}=1}^{h^{\prime \prime}}\left(I_{\alpha^{\prime \prime}}^{\prime \prime}\right)^{*}$. Let $M_{1}, \ldots, M_{u}, N_{1}, \ldots, N_{v}$ be intervals of the first type such that $M_{\xi}^{*}=U_{\xi}, N_{\rho}^{*}=V_{\rho}$ and $\bigcup_{\xi=1}^{u} M_{\xi} \cup \bigcup_{\rho=1}^{v} N_{\rho}=\bigcup_{\alpha=1}^{h} I_{\alpha}$. Define $A^{\prime}=\bigcup_{\xi=1}^{u} M_{\xi}$ and $A^{\prime \prime}=A \backslash A^{\prime}$.

Proposition 6. For $n>0, m>0$ and $h \in \mathbb{N}$ the complete theories of Boolean algebras with elementary characteristic $(h, \omega, 0),(h, \omega, 1),(n, m, 0)$ and $(n, h, 1)$, respectively, have completely many countable models.

Proof. By Propositions 1, 2, 3, 4 and 5.

Proposition 7. The complete theory of Boolean algebras of elementary characteristic $(\omega, 0,0)$ has completely many countable models.

Proof. For any countable graph $G$, let $\mu(G)$ be the linear order $\sum_{h=1}^{\infty} L_{0}^{h} \cdot L(G)$. Note that $\operatorname{ch}(D(1+\mu(G)+1))=(\omega, 0,0)$. It suffices to show that, for $G$ and $G^{\prime}$ countable graphs, $D(1+L(G)+1) \cong D\left(1+L\left(G^{\prime}\right)+1\right)$ iff $D(1+\mu(G)+1) \cong D\left(1+\mu\left(G^{\prime}\right)+1\right)$.

Let $\Phi$ be the graph of an isomorphism from $D(1+L(G)+1)$ onto $D\left(1+L\left(G^{\prime}\right)+1\right)$. We define a V-relation $R \subseteq D(1+\mu(G)+1) \times D\left(1+\mu\left(G^{\prime}\right)+1\right)$.

Every semiclosed interval $[u, v[\subseteq 1+\mu(G)+1$ has the form $[(\alpha, a),(\beta, b)[$, $\left[-\infty,+\infty\left[,\left[-\infty,(\beta, b)\left[\right.\right.\right.\right.$ or $\left[(\alpha, a),+\infty\left[\right.\right.$, where $\alpha, \beta \in L_{0}^{<\omega}$ and $a, b \in L(G)$. Again, 
we can define a projection map $*: D(1+\mu(G)+1) \rightarrow D(1+L(G)+1)$ according to these cases. In the first case, let $\left[u, v\left[^{*}=\left[a, b\left[\right.\right.\right.\right.$ if $a<b$ and put $\left[u, v\left[^{*}=\emptyset\right.\right.$ otherwise. In the second, third and fourth cases let $\left[u, v{ }^{*}\right.$ be $[-\infty,+\infty[,[-\infty, b[,[a,+\infty[$ respectively. Any $A \in D(1+\mu(G)+1)$ can be decomposed into $\bigcup_{k=1}^{\bar{k}} I_{k} \cup \bigcup_{l=1}^{\bar{l}} J_{l}$ $\bar{k}$ where $I_{k}^{*} \neq \emptyset$ and $J_{l}^{*}=\emptyset$. This decomposition is not unique, but $A^{*}=\bigcup_{k=1}^{k} I_{k}^{*}$ is independent of the decomposition (in particular, $\bar{k}$ being 0 is independent of the decomposition). Let $\sigma(A)$ be 1 or 0 depending on whether $A$ contains any atomless element, i.e., $\sigma(A)=1$ iff $A$ contains a subinterval of order type $\eta$. Let $\tau(A) \leq \omega$ be the cardinality of the set of atoms contained in $A$. Notice that if $A^{*} \neq \emptyset$, then $\sigma(A)=1$ and $\tau(A)=\omega$.

For $(A, B) \in D(1+\mu(G)+1) \times D\left(1+\mu\left(G^{\prime}\right)+1\right)$ define

$$
A R B \Leftrightarrow A^{*} \Phi B^{*} \wedge \sigma(A)=\sigma(B) \wedge \tau(A)=\tau(B) .
$$

To see that $R$ is a $V$-relation, the only nontrivial condition to check is the last clause in the definition (and by symmetry, only half of it). For this assume $A R B \dot{\cup} C$. Then we have $A^{*} \Phi(B \cup C)^{*}=B^{*} \cup C^{*}, \sigma(A)=\max (\sigma(A), \sigma(B))$ and $\tau(A)=$ $\tau(B \cup C)=\tau(B)+\tau(C)$. First split $A^{*}$ in two disjoint pieces $A^{\prime}$ and $A^{\prime \prime}$ such that $A^{\prime} \Phi B^{*}$ and $A^{\prime \prime} \Phi C^{*}$. Then a moment reflection shows that it is possible to split correspondingly $A=A_{1} \cup A_{2}$ so that $A_{1}^{*}=A^{\prime}, A_{2}^{*}=A^{\prime \prime}, \sigma\left(A_{1}\right)=\sigma(B)$, $\tau\left(A_{1}\right)=\tau(B), \sigma\left(A_{2}\right)=\sigma(C)$ and $\tau\left(A_{2}\right)=\tau(C)$. This means $A_{1} R B, A_{2} R C$.

Conversely, let $\Psi$ be the graph of an isomorphism $D(1+\mu(G)+1) \rightarrow D(1+$ $\left.\mu\left(G^{\prime}\right)+1\right)$. We define a V-relation $T \subseteq D(1+L(G)+1) \times D\left(1+L\left(G^{\prime}\right)+1\right)$ as follows. For $(A, B) \in D(1+L(G)+1) \times D\left(1+L\left(G^{\prime}\right)+1\right)$ let

$$
\begin{aligned}
A T B \Leftrightarrow & \exists U \in D(1+\mu(G)+1) \exists V \in D\left(1+\mu\left(G^{\prime}\right)+1\right) \\
& \left(U^{*}=A \wedge V^{*}=B \wedge U \Psi V\right) .
\end{aligned}
$$

For $U \in D(1+\mu(G)+1)$ we have $U^{*}=\emptyset$ iff $(\Psi(U))^{*}=\emptyset$, since $U^{*}=\emptyset$ means that $U$ belongs to some iteration of the Ershov-Tarski ideal, so $A T \emptyset \Rightarrow A=\emptyset$ and $\emptyset T B \Rightarrow B=\emptyset$.

Next assume $A T B \cup \dot{\cup}$ and suppose $B \neq \emptyset \neq C$ (if either $B$ or $C$ is empty, then it will do to simply split $A=A \dot{\cup} \emptyset$ or $A=\emptyset \dot{\cup} A)$. Let $U \in D(1+\mu(G)+1)$ and $V \in D\left(1+\mu\left(G^{\prime}\right)+1\right)$ be such that $U^{*}=A, V^{*}=B \cup C$ and $U \Psi V$. Let $W, Z \in D\left(1+\mu\left(G^{\prime}\right)+1\right)$ be such that $V=W \dot{\cup} Z, W^{*}=B$ and $Z^{*}=C$. Then split $U=X \dot{\cup} Y$ in such a way that $X \Psi W$ and $Y \Psi Z$. Thus we have $X^{*} \dot{\cup} Y^{*}=A$, $X^{*} T B$ and $Y^{*} T C$.

Proposition 8. For $m \in \mathbb{N}$ the complete theories of Boolean algebras with elementary characteristic $(0, m, 0)$ and $(0, m, 1)$ respectively have only one model, up to isomorphism, of cardinality less than or equal to $\aleph_{0}$.

Proof. The Boolean algebras with elementary characteristic $(0, m, 0)$ are the finite ones with $m$ atoms. For characteristic $(0, m, 1)$ we get the cartesian products of a finite Boolean algebra with $m$ atoms and an atomless countable Boolean algebra. 


\section{Applications to some algebraic AND TOPOLOGICAL EQUIVALENCE RELATIONS}

In this section we prove the Borel completeness of several other equivalence relations arising in algebra and topology.

We first deal with the translation relation between closed subsets of the Cantor space. We actually obtain a stronger result by concentrating on nowhere dense closed subsets. Let

$$
\mathcal{S}=\left\{K \in \mathcal{K}\left(2^{\mathbb{N}}\right) \mid K \text { is nowhere dense }\right\} .
$$

Then $\mathcal{S}$ is a Borel subset of $\mathcal{K}\left(2^{\mathbb{N}}\right)$, thus is itself a standard Borel space. We define an equivalence relation $\cong *$ on $\mathcal{S}$ as follows:

$$
K_{1} \cong{ }^{*} K_{2} \text { iff } \exists \pi \in \operatorname{Aut}\left(2^{\mathbb{N}}\right)\left(\pi^{*} K_{1}=K_{2}\right) .
$$

Then $\cong *$ is in fact an orbit equivalence relation induced by a Borel action of $\operatorname{Aut}\left(2^{\mathbb{N}}\right)$ on $\mathcal{S}$. By the completeness of $C_{\infty}$, we have $\cong{ }^{*}{ }_{B} C_{\infty}$.

Theorem 3. The equivalence relation $\cong *$ between nowhere dense closed subsets of the Cantor space $2^{\mathbb{N}}$ is Borel complete.

Proof. It suffices to define a Borel reduction $\theta$ from the homeomorphism relation $\cong$ on $\mathcal{K}\left(2^{\mathbb{N}}\right)$ to $\cong *$. Let $K \in \mathcal{K}\left(2^{\mathbb{N}}\right)$ and $T_{K}$ be the pruned subtree of $2^{<\mathbb{N}}$ whose infinite branches are exactly the elements of $K$. Let $h: 2^{<\mathbb{N}} \rightarrow 2^{<\mathbb{N}}$ be a mapping defined inductively as follows: $h(\emptyset)=\emptyset$ and $h\left(t^{\frown} n\right)=h(t)^{\frown} n^{\frown} 0$. Let $T_{K}^{\prime}$ be the pruned subtree of $2^{<\mathbb{N}}$ generated by the set $\left\{h(t) \mid t \in T_{K}\right\}$. Finally, let $\theta(K)$ be the set of infinite branches of $T_{K}^{\prime}$. Now it is obvious that $\theta(K)$ is nowhere dense. Moreover, $\theta$ is in fact a continuous injection from $\mathcal{K}\left(2^{\mathbb{N}}\right)$ into $\mathcal{K}\left(2^{\mathbb{N}}\right)$, thus a Borel function from $\mathcal{K}\left(2^{\mathbb{N}}\right)$ into $\mathcal{S}$.

The mapping $h$ induces a homeomorphism from $2^{\mathbb{N}}$ onto some nowhere dense closed subset of $2^{\mathbb{N}}$, which we also denote by $h$. Then in fact, for any $K \in \mathcal{K}\left(2^{\mathbb{N}}\right)$, we have $\theta(K)=h^{\text {" } K} K$. Now for $K_{1}, K_{2} \in \mathcal{K}\left(2^{\mathbb{N}}\right)$, if $\theta\left(K_{1}\right) \cong * \theta\left(K_{2}\right)$ via $\pi \in \operatorname{Aut}\left(2^{\mathbb{N}}\right)$, then $K_{1} \cong K_{2}$ via $h^{-1} \circ \pi \circ h$. This is the backward direction of the reduction. For the forward direction, if $K_{1} \cong K_{2}$, then we know that there is a homeomorphism between $\theta\left(K_{1}\right)$ and $\theta\left(K_{2}\right)$. The proof will be complete granting the following general lemma.

Lemma 1 (folklore). Let $K_{1}$ and $K_{2}$ be nowhere dense closed subsets of the Cantor space $2^{\mathbb{N}}$. Let $f$ be a homeomorphism between $K_{1}$ and $K_{2}$. Then $f$ can be extended to an autohomeomorphism of the whole space $2^{\mathbb{N}}$, i.e., there is $F \in \operatorname{Aut}\left(2^{\mathbb{N}}\right)$ such that $F \uparrow K_{1}=f$.

Proof. First we fix some notation. For any tree $\mathcal{T} \subseteq 2^{<\mathbb{N}}$ let $[\mathcal{T}]$ denote the set of all infinite branches of $\mathcal{T}$. If $t \in \mathcal{T}$, let $\mathcal{T}_{t}=\{s \in \mathcal{T} \mid s \subseteq t$ or $t \subseteq s\}$, then $\mathcal{T}_{t}$ is a subtree of $\mathcal{T}$ and $\left[\mathcal{T}_{t}\right] \subseteq[\mathcal{T}]$. If $\tau$ is a finite subset of $\mathcal{T}$, then let $\mathcal{T}_{\tau}=\bigcup_{t \in \tau} \mathcal{T}_{t}$.

Now let $K_{1}$ and $K_{2}$ be nowhere dense closed subsets of $2^{\mathbb{N}}$. Let $\mathcal{T}^{0}, \mathcal{T}^{1}$ and $\mathcal{T}^{2}$ be pruned subtrees of $2^{<\mathbb{N}}$ with $\left[\mathcal{T}^{0}\right]=2^{\mathbb{N}},\left[\mathcal{T}^{1}\right]=K_{1}$ and $\left[\mathcal{T}^{2}\right]=K_{2}$. Let $d, d_{1}$ and $d_{2}$ be compatible complete metrics on $2^{\mathbb{N}}, K_{1}$ and $K_{2}$, respectively. For any $K \in$ $\mathcal{K}\left(2^{\mathbb{N}}\right)$, we use $d(K)$ to denote the diameter of $K$, i.e., $d(K)=\sup \{d(x, y) \mid x, y \in$ $K\}$. Similarly, $d_{1}$ and $d_{2}$ will also be used to denote the diameters of closed subsets of $K_{1}$ and $K_{2}$, respectively.

Now let $f$ be a homeomorphism from $K_{1}$ onto $K_{2}$. Using the hypothesis that $K_{1}$ and $K_{2}$ are nowhere dense, we can obtain sequences $\left\{W_{i}^{n}\right\},\left\{\tau_{i}^{n}\right\}$ and $\left\{\sigma_{i}^{n}\right\}$ 
for $n \in \mathbb{N}$ and $i<N(n)$ for some $N(n) \in \mathbb{N}$, so that the following conditions are fulfilled:

(1) $N(0)=1, W_{0}^{0}=K_{1}, \tau_{0}^{0}=\sigma_{0}^{0}=\{\emptyset\}$;

(2) for each $n,\left\{W_{i}^{n}\right\}_{i<N(n)}$ is a partition of $K_{1}$ into disjoint relatively clopen sets;

(3) for each $n$, the partition $\left\{W_{j}^{n+1}\right\}_{j<N(n+1)}$ is a refinement of the partition $\left\{W_{i}^{n}\right\}_{i<N(n)}$

(4) for each $n$ and $i, d_{1}\left(W_{i}^{n}\right)<2^{-i}$ and $d_{2}\left(f^{\text {" }} W_{i}^{n}\right)<2^{-i}$;

(5) for each $n$ and $i, \tau_{i}^{n}$ is a finite set of pairwise incomparable elements of $\mathcal{T}^{1}$ such that $W_{i}^{n}=\left[\mathcal{T}_{\tau_{i}^{n}}^{1}\right]$, and $\sigma_{i}^{n}$ is a finite set of pairwise incomparable elements of $\mathcal{T}^{2}$ such that $f^{\text {" }} W_{i}^{n}=\left[\mathcal{T}_{\sigma_{i}^{n}}^{2}\right]$;

(6) if $W_{j}^{n+1} \subseteq W_{i}^{n}$, then $\forall t \in \tau_{j}^{n+1} \exists s \in \tau_{i}^{n}(s \subseteq t)$ and $\forall t \in \sigma_{j}^{n+1} \exists s \in \sigma_{i}^{n}(s \subseteq t)$;

(7) for each $n$ and $i$,

$$
U_{i}^{n}={ }_{\operatorname{def}}\left[\mathcal{T}_{\tau_{i}^{n}}^{0}\right] \backslash \bigcup\left\{\left[\mathcal{T}_{\tau_{j}^{n+1}}^{0}\right] \mid W_{j}^{n+1} \subseteq W_{i}^{n}\right\} \neq \emptyset,
$$

and

$$
V_{i}^{n}=_{\operatorname{def}}\left[\mathcal{T}_{\sigma_{i}^{n}}^{0}\right] \backslash \bigcup\left\{\left[\mathcal{T}_{\sigma_{j}^{n+1}}^{0}\right] \mid W_{j}^{n+1} \subseteq W_{i}^{n}\right\} \neq \emptyset
$$

Now we have that $\left\{K_{1}\right\} \cup\left\{U_{i}^{n}\right\}_{n \in \mathbb{N}, i<N(n)}$ and $\left\{K_{2}\right\} \cup\left\{V_{i}^{n}\right\}_{n \in \mathbb{N}, i<N(n)}$ are both decompositions of $2^{\mathbb{N}}$. Moreover, each $U_{i}^{n}$ or $V_{i}^{n}$ is a nonempty clopen subset of $2^{\mathbb{N}}$, hence homeomorphic to $2^{\mathbb{N}}$. Now $F$ can be defined by the union of $f$ together with homeomorphisms between all the pairs $U_{i}^{n}$ and $V_{i}^{n}$.

We learned the lemma from S. Solecki. The above proof of the lemma is a modification of Solecki's argument. (A reference for the statement without proof is vM89, Exercise 6.1.11].) In fact, this proof is slightly more complicated than we need; the reason we present it this way is that we are going to use a variant of it below to obtain a stronger result, where the proof can be reused.

We now turn to an algebraic equivalence relation. Consider the expansion $\mathcal{L}^{\prime}$ of the language $\mathcal{L}$ of Boolean algebras by a unary relation symbol. Structures of $\mathcal{L}^{\prime}$ are pairs $(B, I)$. We consider the countable models $(B, I)$ where $B$ is a Boolean algebra and $I$ is an ideal of $B$. It follows trivially from our main theorem that the isomorphism relation between all such pairs is Borel complete. However, if we fix a particular Boolean algebra $B$ and consider the restricted isomorphism relation between the pairs $(B, I)$, the equivalence relation need not be Borel complete. In particular, it is very easy to come up with a superatomic $B$ so that the corresponding equivalence relation is not Borel complete. Nevertheless, we show that if we take the countable atomless Boolean algebra $B_{\eta}$, the corresponding isomorphism relation is indeed Borel complete.

Theorem 4. The isomorphism relation between pairs $\left(B_{\eta}, I\right)$, where $I$ is an ideal of $B_{\eta}$, is Borel complete.

Proof. We fix an arbitrary realization of $B_{\eta}$ as an element of $\operatorname{Mod}\left(T_{\mathrm{BA}}\right)$. By the remark in section 2 in which we constructed an alternative reduction from CBA to $\mathcal{K}\left(2^{\mathbb{N}}\right)$, we have a perfect generating tree $\mathcal{T} \subset B_{\eta}$ which is isomorphic to $2^{<\mathbb{N}}$. For the rest of the proof we do not distinguish $\mathcal{T}$ and $2^{<\mathbb{N}}$.

Now for each nowhere dense closed subset $K$ of $2^{\mathbb{N}}$, define the ideal $I_{K}$ to be the ideal of $B_{\eta}$ generated by the subset $\{t \in \mathcal{T} \mid \forall x \in K(t \nsubseteq x)\}$. Now if $K_{1} \cong{ }^{*} K_{2}$ 
via $f \in \operatorname{Aut}\left(2^{\mathbb{N}}\right)$, then by the same remark again, $f$ induces an automorphism $\pi$ of $B_{\eta}$ with $\pi$ " $I_{K_{1}}=I_{K_{2}}$. Conversely, if $\pi \in \operatorname{Aut}\left(B_{\eta}\right)$ witnesses $\left(B_{\eta}, I_{K_{1}}\right) \cong\left(B_{\eta}, I_{K_{2}}\right)$, then $\pi$ " $I_{K_{1}}=I_{K_{2}}$. Moreover, $\pi$ induces an $f \in \operatorname{Aut}\left(2^{\mathbb{N}}\right)$ such that $f$ " $K_{1}=K_{2}$.

Finally, the assignment $K \mapsto I_{K}$ is a Borel function, since any element of $B_{\eta}$ determines a finite subset of $\mathcal{T}$, which in turn determines a clopen subset of $2^{\mathbb{N}}$; and the element of $B_{\eta}$ is in $I_{K}$ iff $K$ is disjoint from this clopen set.

We now consider another expansion $\mathcal{L}^{\prime \prime}$ of the language of Boolean algebras by adding to it a unary function symbol. Consider the models of $\mathcal{L}^{\prime \prime}$ which are pairs $(B, \varphi)$, where $B$ is a Boolean algebra and $\varphi$ is an endomorphism or even an automorphism of $B$. Again, the general isomorphism relation between such pairs is Borel complete, but for specific $B$ the restricted isomorphism relation is not necessarily complete. We again consider the problem in which the Boolean algebra is fixed as $B_{\eta}$. This turns out to be related to the classification problem of the conjugacy classes of $\operatorname{Aut}\left(2^{\mathbb{N}}\right)$, which we define below.

Let $\operatorname{Aut}\left(2^{\mathbb{N}}\right)$ act on itself by conjugacy, i.e., $h . g=h \circ g \circ h^{-1}$. This is a continuous action. We denote the orbit equivalence relation by $\sim$ and call it the conjugacy equivalence relation of Aut $\left(2^{\mathbb{N}}\right)$. In general, Aut $\left(2^{\mathbb{N}}\right)$ can act on the space of all continuous functions from $2^{\mathbb{N}}$ into $2^{\mathbb{N}}$, which we denote by $C\left(2^{\mathbb{N}}, 2^{\mathbb{N}}\right)$, in the same manner. We call the resulting orbit equivalence relation the conjugacy equivalence relation of $C\left(2^{\mathbb{N}}, 2^{\mathbb{N}}\right)$ and denote it by $\sim$ also, if there is no danger of confusion. Since these are orbit equivalence relations induced by continuous actions of $\operatorname{Aut}\left(2^{\mathbb{N}}\right)$ on Polish spaces, we know that they are Borel reducible to $C_{\infty}$.

Theorem 5. The following equivalence relations are all Borel complete:

(i) the conjugacy equivalence relation of $\operatorname{Aut}\left(2^{\mathbb{N}}\right)$;

(ii) the conjugacy equivalence relation of $C\left(2^{\mathbb{N}}, 2^{\mathbb{N}}\right) \backslash \operatorname{Aut}\left(2^{\mathbb{N}}\right)$;

(iii) the conjugacy equivalence relation of $C\left(2^{\mathbb{N}}, 2^{\mathbb{N}}\right)$;

(iv) the isomorphism relation between pairs $\left(B_{\eta}, \varphi\right)$, where $\varphi$ is an automorphism of $B_{\eta}$.

(v) the isomorphism relation between pairs $\left(B_{\eta}, \varphi\right)$, where $\varphi$ is an endomorphism but non-automorphism of $B_{\eta}$;

(vi) the isomorphism relation between pairs $\left(B_{\eta}, \varphi\right)$, where $\varphi$ is an endomorphism of $B_{\eta}$.

Proof. Recall the remark we made in section 2 that $\operatorname{Aut}\left(2^{\mathbb{N}}\right)$ and $\operatorname{Aut}\left(B_{\eta}\right)$ are isomorphic Polish groups. It follows immediately that (i) $\Leftrightarrow$ (iv). Also, it is obvious that (i) $\Rightarrow$ (iii) since the space Aut $\left(2^{\mathbb{N}}\right)$ is an invariant Borel subspace of $C\left(2^{\mathbb{N}}, 2^{\mathbb{N}}\right)$. For a similar reason, (iv) $\Rightarrow$ (vi). We complete the proof by showing (i) and the implications (i) $\Rightarrow$ (ii) and (iv) $\Rightarrow$ (v).

We show (i) by reducing to it the equivalence $\cong *$ between nowhere dense closed subsets of $2^{\mathbb{N}}$. For this we use the notation defined in the proof of Theorem 3. For each $K \in \mathcal{S}$, define an automorphism $f_{K} \in \operatorname{Aut}\left(2^{\mathbb{N}}\right)$ as follows. If $x \in K$, then let $f_{K}(x)=x$. If $x \notin K$ and let $\mathcal{T}$ be the subtree of $2^{<\mathbb{N}}$ with $[\mathcal{T}]=K$, then there is $s \in 2^{<\mathbb{N}}$ such that $s \subseteq x, s \notin \mathcal{T}$ and $t \in \mathcal{T}$ forall $t \subset s$. Write $x=s^{\curlyvee} b^{\curlyvee} y$, where $b \in\{0,1\}$ and $y \in 2^{\mathbb{N}}$. Then set $f_{K}(x)=s^{\frown}(1-b)^{\frown} y$. It is easy to see that $f_{K} \in \operatorname{Aut}\left(2^{\mathbb{N}}\right)$. Moreover, $K$ is exactly the set of fixed points of $f_{K}$. Now suppose $K_{1}, K_{2} \in \mathcal{S}$. If there is some $h \in \operatorname{Aut}\left(2^{\mathbb{N}}\right)$ such that $f_{K_{2}}=h \circ f_{K_{1}} \circ h^{-1}$, then $h^{\text {" }} K_{1}=K_{2}$, hence $K_{1} \cong{ }^{*} K_{2}$. On the other hand, suppose that $K_{1} \cong{ }^{*} K_{2}$, then in particular there is a homeomorphism $f$ between $K_{1}$ and $K_{2}$. It suffices to show that 
$f$ can be extended to an $F \in \operatorname{Aut}\left(2^{\mathbb{N}}\right)$ such that $f_{K_{2}}=F \circ f_{K_{1}} \circ F^{-1}$. For this we use the same proof of Lemma 1 again. Suppose $\mathcal{T}^{0}, \mathcal{T}^{1}, \mathcal{T}^{2},\left\{W_{i}^{n}\right\},\left\{\tau_{i}^{n}\right\},\left\{\sigma_{i}^{n}\right\},\left\{U_{i}^{n}\right\}$ and $\left\{V_{i}^{n}\right\}$ are defined as in the proof of Lemma 1. F will still be defined by the union of $f$ and some homeomorphism between the pairs $\left(U_{i}^{n}, V_{i}^{n}\right)$. Let us fix some $n$ and $i$. For notational simplicity, denote by $U$ and $V$ the corresponding $U_{i}^{n}$ and $V_{i}^{n}$, respectively. Let $C_{j}=\left\{s \in 2^{<\mathbb{N}} \mid s \notin \mathcal{T}^{j}\right.$ but $t \in \mathcal{T}^{j}$ for all $\left.t \subset s\right\}$ for $j=1,2$. Then there are a finite number of pairwise incompatible elements $s_{0}, \ldots, s_{k} \in C_{1}$ and $t_{0}, \ldots, t_{l} \in C_{2}$ such that $U=\left[\mathcal{T}_{s_{0}}^{0}\right] \cup \cdots \cup\left[\mathcal{T}_{s_{k}}^{0}\right]$ and $V=\left[\mathcal{T}_{t_{0}}^{0}\right] \cup \cdots \cup\left[\mathcal{T}_{t_{l}}^{0}\right]$, where the unions are disjoint. Decompositions of $U$ and $V$ in this form are in fact unique.

Note that for each $i \leq k,\left[\mathcal{T}_{s_{i}}^{0}\right]$ is invariant under $f_{K_{1}}$; specifically, for each $x \in\left[\mathcal{T}_{s_{i}}^{0}\right]$, say $x=s_{i}{ }^{\frown} b^{\frown} y$ for $b \in\{0,1\}$ and $y \in 2^{\mathbb{N}}, f_{K_{1}}(x)=s_{i}{ }^{\curlyvee}(1-b)^{\frown} y$. Similarly, for each $j \leq l,\left[\mathcal{T}_{t_{j}}^{0}\right]$ is invariant under $f_{K_{2}}$ in the same manner. To define the homeomorphism $F$ between $U$ and $V$, we can assume, without loss of generality, that $k=0$. If $l=0$, then let $F\left(s_{0} \wedge y\right)=t_{0} \wedge y$ for any $y \in 2^{\mathbb{N}}$ and we are done. If $l>0$, then first find a set of pairwise imcompatible elements $u_{0}, \ldots, u_{l} \in 2^{<\mathbb{N}}$ such that $\left[\mathcal{T}_{\left\{s_{0} \frown 0^{-} u_{0}, \ldots, s_{0} \frown 0^{-} u_{l}\right\}}^{0}\right]=\left[\mathcal{T}_{s_{0}-0}^{0}\right] . F$ is then defined by

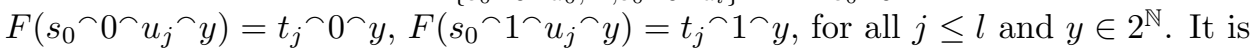
easy to check that $F$ works. This finishes the proof of (i).

To see that (i) $\Rightarrow$ (ii), we define a Borel reduction $\theta: \operatorname{Aut}\left(2^{\mathbb{N}}\right) \rightarrow C\left(2^{\mathbb{N}}, 2^{\mathbb{N}}\right) \backslash$ $\operatorname{Aut}\left(2^{\mathbb{N}}\right)$ as follows. For $f \in \operatorname{Aut}\left(2^{\mathbb{N}}\right)$, let $\theta(f)\left(0^{\frown} x\right)=0^{\frown} f(x)$ and $\theta(f)\left(1^{\frown} x\right)=$ $1^{\frown} 0^{\frown} x$, for all $x \in 2^{\mathbb{N}}$. Let $f, g \in \operatorname{Aut}\left(2^{\mathbb{N}}\right)$. If $h \in \operatorname{Aut}\left(2^{\mathbb{N}}\right)$ is such that $g=h \circ f \circ h^{-1}$, then letting $H\left(0^{\frown} x\right)=0^{\frown} h(x)$ and $H\left(1^{\frown} x\right)=1^{\frown} x$ for all $x \in 2^{\mathbb{N}}$, we have that $\theta(g)=H \circ \theta(f) \circ H^{-1}$.

On the other hand, suppose $\theta(g)=H \circ \theta(f) \circ H^{-1}$ for some $H \in \operatorname{Aut}\left(2^{\mathbb{N}}\right)$. Let $B=\left\{0^{-} x \mid x \in 2^{\mathbb{N}}\right\}$ and $\beta$ be the canonical homeomorphism from $2^{\mathbb{N}}$ onto $B$. Let $C=2^{\mathbb{N}} \backslash B$ and $\gamma$ be the canonical homeomorphism from $2^{\mathbb{N}}$ onto $C$. Let $B_{0}=H$ " $B \cap B, B_{1}=B \backslash B_{0}$ and $D_{0}=\beta^{-1 " ~} B_{0}, D_{1}=2^{\mathbb{N}} \backslash D_{0}=\beta^{-1 \text { " }} B_{1}$. Then define

$$
h(x)= \begin{cases}\beta^{-1}(H(\beta(x))) & \text { if } x \in D_{0}, \\ \beta^{-1}\left(H^{2}(\beta(x))\right) & \text { if } x \in D_{1} .\end{cases}
$$

We check that $h \in \operatorname{Aut}\left(2^{\mathbb{N}}\right)$ with $g=h \circ f \circ h^{-1}$. First, note that $H$ " $B_{0}=B_{0}$. Therefore, for any $x \in D_{0}, h(x) \in D_{0}$ and

$$
\begin{aligned}
g(x) & =\beta^{-1}(\theta(g)(\beta(x))) \\
& =\beta^{-1}\left(H\left(\theta(f)\left(H^{-1}(\beta(x))\right)\right)\right) \\
& =\beta^{-1}\left(H\left(\theta(f)\left(\beta\left(\beta^{-1}\left(H^{-1}(\beta(x))\right)\right)\right)\right)\right) \\
& =\beta^{-1}\left(H\left(\theta(f)\left(\beta\left(h^{-1}(x)\right)\right)\right)\right) \\
& =\beta^{-1}\left(H\left(\beta\left(\beta^{-1}\left(\theta(f)\left(\beta\left(h^{-1}(x)\right)\right)\right)\right)\right)\right) \\
& =\beta^{-1}\left(H\left(\beta\left(f\left(h^{-1}(x)\right)\right)\right)\right) \\
& =h\left(f\left(h^{-1}(x)\right)\right) .
\end{aligned}
$$

For $x \in D_{1}$, we have

$$
\begin{aligned}
g(x) & =\beta^{-1}\left(H\left(\theta(f)\left(H^{-1}(\beta(x))\right)\right)\right) \\
& =\beta^{-1}\left(H\left(1^{\frown} 0^{\frown} \gamma^{-1}\left(H^{-1}(\beta(x))\right)\right)\right)
\end{aligned}
$$

since $H^{-1}(\beta(x)) \in C$, and

$$
\begin{aligned}
f(x) & =\beta^{-1}\left(H^{-1}(\theta(g)(H(\beta(x))))\right) \\
& =\beta^{-1}\left(H^{-1}\left(1^{\frown} 0^{-} \gamma^{-1}(H(\beta(x)))\right)\right)
\end{aligned}
$$


since $H(\beta(x)) \in C$. Therefore, for $x \in D_{1}$, the definition of $h$ gives that $g(x)=$ $h\left(f\left(h^{-1}(x)\right)\right)$. The computations also imply that $h$ is well-defined as an automorphism of $2^{\mathbb{N}}$. Thus we have verified the implication (i) $\Rightarrow$ (ii).

The proof for (iv) $\Rightarrow$ (v) uses a similar idea as the above proof. Specifically, let $a, b \in B_{\eta}$ be such that $b<C(a)$ and fix an isomorphism $\delta$ from $\widehat{C(a)}$ onto $\hat{b}$. Also, fix an isomorphism $\beta$ from $B_{\eta}$ onto $\hat{a}$. Then define a Borel reduction $\theta$ by, given any $\alpha \in \operatorname{Aut}\left(B_{\eta}\right)$ and $x \in B_{\eta}$,

$$
\theta(\alpha)(x)=\beta\left(\alpha\left(\beta^{-1}(x \cap a)\right)\right) \cup \gamma(x \cap C(a)) .
$$

Then a similar argument as above shows that $\theta$ is a reduction.

In the above theorem the completeness of (ii) emphasizes that the class in (iii) may consist of many complete parts other than the one corresponding to (i). Furthermore, the function in $C\left(2^{\mathbb{N}}, 2^{\mathbb{N}}\right)$ we constructed in the proof is in fact a topological embedding from $2^{\mathbb{N}}$ into $2^{\mathbb{N}}$ (but not onto). The proof could be slightly simplified by using a function which is not one-one. Similar remarks apply also to the second half of the theorem. Note also, however, that (iii) is not a topological version of (vi), since the generating tree argument does not give a topological isomorphism between spaces $C\left(2^{\mathbb{N}}, 2^{\mathbb{N}}\right)$ and that of all endomorphisms of $B_{\eta}$.

The conjugacy equivalence relation on $\operatorname{Aut}\left(2^{\mathbb{N}}\right)$ was considered in a different form by Anderson ([An58]), who showed that there are $2^{\aleph_{0}}$ many equivalence classes. Our result can be viewed as an improvement.

In the rest of this section we give an analysis for the uniform Boolean algebras used in Ketonen's classification of countable Boolean algebras ([Ket78). A clearer account of Ketonen's results can be found in Pi89.

For our purpose it suffices to summarize the results as the statement: the following equivalence relations are bi-reducible to each other:

- the isomorphism relation for countable uniform Boolean algebras;

- the homeomorphism relation for separable uniform Boolean spaces;

- the relation of equivalence for upper semicontinuous mappings of the Cantor set to $\omega_{1}$

- the relation of equivalence for additive functions of $B_{\eta}$ to $\omega_{1}$.

The rank functions on the Cantor set or the additive functions on $B_{\eta}$ naturally suggest a hierarchy of uniform Boolean spaces or of uniform Boolean algebras into $\aleph_{1}$ many classes. It is possible to equip these classes with a standard Borel structure so that the witnesses of the bi-reducibility become Borel. Below we focus on the last equivalence relation mentioned above and give some details of the coding. The situations with the other equivalence relations are completely parallel.

For simplicity, we will use a modified notion of additive functions.

Definition. Let $B$ be a countable Boolean algebra. A function $\sigma$ is called an additive function of $B$ if $\sigma: B \rightarrow \omega_{1}$ satisfies that $\sigma\left(0_{B}\right)=0$ and for any $a, b \in B$, $\sigma(a \cup b)=\max \{\sigma(a), \sigma(b)\}$. We call the countable ordinal sup range $(\sigma)=\sigma\left(1_{B}\right)$ the height of $\sigma$. For each countable ordinal $\alpha>0$, define

$$
\mathcal{R}_{\alpha}=\left\{\sigma \mid \sigma \text { is an additive function of } B_{\eta} \text { of height } \alpha\right\} .
$$

Fixing a realization of $B_{\eta}$ as an element of $\operatorname{Mod}\left(T_{\mathrm{BA}}\right)$ and for each $\alpha>0$ fixing a bijection between $\alpha$ and some ordinal $\leq \omega$, the space $\mathcal{R}_{\alpha}$ can be viewed as a Borel subset of the Baire space $\mathbb{N}^{\mathbb{N}}$, hence it is a standard Borel space. Note that if $\alpha \neq \beta$ 
are nonzero countable ordinals, then $\mathcal{R}_{\alpha} \cap \mathcal{R}_{\beta}=\emptyset$. Define $\mathcal{R}=\bigcup_{\alpha<\omega_{1}} \mathcal{R}_{\alpha}$. Then $\mathcal{R}$ is the collection of all additive functions of $B_{\eta}$ to $\omega_{1}$.

For $\sigma, \tau \in \mathcal{R}$, define the equivalence relation

$$
\sigma \cong \tau \text { iff } \tau=\sigma \circ \pi \text { for some } \pi \in \operatorname{Aut}\left(B_{\eta}\right) .
$$

Then on each $\mathcal{R}_{\alpha} \cong$ is the orbit equivalence relation of a Borel action of $\operatorname{Aut}\left(B_{\eta}\right)$; moreover, $\mathcal{R}_{\alpha}$ is an invariant subspace of $\mathcal{R}$.

The following theorem shows that the decomposition of all uniform Boolean algebras into $\aleph_{1}$ many classes does not essentially simplify the classification problem.

Theorem 6. For each countable ordinal $\alpha>0$, the equivalence relation $\cong$ on $\mathcal{R}_{\alpha}$ is Borel complete.

Proof. For $\alpha=1$ we define a Borel reduction from the pairs $\left(B_{\eta}, I\right)$, where $I$ is a nontrivial ideal of $B_{\eta}$. It then follows from Theorem 4 that $\cong$ on $\mathcal{R}_{1}$ is Borel complete. For each nontrivial ideal $I$ of $B_{\eta}$, define an additive function $\sigma_{I}$ by

$$
\sigma_{I}(x)= \begin{cases}0 & \text { if } x \in I, \\ 1 & \text { otherwise. }\end{cases}
$$

Then $\left(B_{\eta}, I\right) \mapsto \sigma_{I}$ is a Borel reduction, as required.

In general, if $\alpha>1$, then we define a Borel reduction from $\mathcal{R}_{1}$ to $\mathcal{R}_{\alpha}$. For $\sigma \in \mathcal{R}_{1}$, define $\sigma^{\prime}(x)=\alpha \cdot \sigma(x)$ for all $x \in B_{\eta}$. Then $\sigma \mapsto \sigma^{\prime}$ is the required reduction.

It might be amusing for the reader to see what kind of uniform Boolean algebras correspond to additive functions of height 1 on $B_{\eta}$. These are exactly the Boolean algebras $B$ such that $B / \mathcal{F}(B)$ is atomless, where $\mathcal{F}(B)$ is the Frechét ideal of $B$. It follows from Theorem 6 that there are completely many non-isomorphic such Boolean algebras.

\section{Some applications to the theory of $C^{*}$-Algebras}

We are now going to use results from the preceding sections and apply them, together with some classical constructions, to equivalence relations arising in the theory of $C^{*}$-algebras. We refer to $\mathrm{Mu}$ 90 or to [B198] for the basic definitions. We would like to thank A. Marcone and G. Panti for having brought these applications to our attention.

First note that the concept of Borelness also makes sense for equivalence relations defined on analytic subspaces of standard Borel spaces. For such equivalence relations we will also talk about Borel reductions and Borel completeness. This will simplify our notation in the rest of the section.

Following [Kec98] we consider the standard Borel space of codes for separable $C^{*}$-algebras, as follows. Fix an infinite dimensional Hilbert space $H$ and let $\mathcal{B}(H)$ be the $C^{*}$-algebra of bounded operators on $H$, endowed with the Borel structure induced by the weak topology (which is the same as the Borel structure induced by the $\sigma$-weak, strong and $\sigma$-strong topologies). Every separable $C^{*}$-algebra is isomorphic to a $C^{*}$-subalgebra of $\mathcal{B}(H)$. Let $\Gamma=(\mathcal{B}(H))^{\mathbb{N}}$. Then each element $\gamma \in \Gamma$ codes a separable $C^{*}$-algebra, namely the one generated by $\{\gamma(n)\}_{n \in \mathbb{N}}$.

Recall that a $C^{*}$-algebra is called approximately finite-dimensional (or $A F$ ) if it is the inductive limit of finite dimensional $C^{*}$-subalgebras. AF algebras were first introduced and extensively studied by $\mathrm{O}$. Bratteli in [Br72]. We denote by AF the 
class of AF algebras. It can be shown that it is an analytic subset of $\Gamma$; though it is not known whether it is Borel (see Kec98).

Let $\varphi_{1}: \mathcal{K}\left(E_{1 / 3}\right) \rightarrow \Gamma$ be the function assigning to each $K \in \mathcal{K}\left(E_{1 / 3}\right)$ a code for $\mathcal{C}(K)={ }_{\text {def }} \mathcal{C}(K, \mathbb{C})$, the algebra of continuous functions from $K$ to $\mathbb{C}$. The function $\varphi_{1}$ can be defined to be Borel, by suitably choosing a countable dense set in $\mathcal{C}(K)$ (e.g., polynomials with coefficients of the form $p+\mathrm{i} q$, with $p, q \in \mathbb{Q}$ ) and using the Gelfand-Naimark-Segal representation.

We consider the isomorphism relation on $\Gamma$, that is, two elements $\gamma, \gamma^{\prime}$ of $\Gamma$ are isomorphic iff they code isomorphic separable $C^{*}$-algebras. Then the function $\varphi_{1}$ is a Borel reduction from the homeomorphism relation on $\mathcal{K}\left(E_{1 / 3}\right)$ to the isomorphism relation on $\Gamma$. This follows from the classical result that, if $K$ and $K^{\prime}$ are nonhomeomorphic compact spaces, then $\mathcal{C}(K)$ and $\mathcal{C}\left(K^{\prime}\right)$ are non-isomorphic Banach algebras (see, e.g., [Na72, page 196]).

In effect we have shown that the isomorphism relation between separable $C^{*}$ algebras is Borel complete. Moreover, the values taken by the function $\varphi_{1}$ are commutative AF algebras. We have thus proved the following result.

Theorem 7. The isomorphism relation for commutative AF algebras is Borel complete.

From this we have some immediate corollaries.

Corollary 8. The isomorphism relation for commutative AF algebras is $\boldsymbol{\Sigma}_{1}^{1}$ complete (as an analytic subset of $\mathrm{AF} \times \mathrm{AF}$ ); in particular, it is not Borel.

Corollary 9. The isomorphism relation for AF algebras is Borel complete.

The result can be propagated to other kinds of structures by the classical classifications of AF algebras. Recall that, to every AF algebra $A$ a scaled ordered group $\left(K_{0}(A), K_{0}(A)_{+},\left[1_{A}\right]\right)$, called the dimension group, can be associated, so that $\mathrm{AF}$ algebras $A$ and $B$ are isomorphic iff their dimension groups are. Dimension groups form a class of countable structures, which we denote by DG. This is a Borel class, since it can be described as the class of countable ordered groups which are unperforated and satisfy the Riesz interpolation property. Consider the isomorphism relation on DG. Defining a function $\varphi_{2}: \mathcal{K}\left(2^{\mathbb{N}}\right) \rightarrow$ DG by assigning to each $K \in \mathcal{K}\left(2^{\mathbb{N}}\right)$ the dimension group of $\mathcal{C}(K), \varphi_{2}$ is a Borel reduction from the homeomorphism relation on $\mathcal{K}\left(2^{\mathbb{N}}\right)$ to the isomorphism relation on DG. Hence we have the following result.

Theorem 10. The class of dimension groups is Borel complete.

Finally, we consider Bratteli diagrams. For each AF algebra $A$ the Bratteli diagram $D(A)$ of $A$ is a combinatorial way to code all the information about the structure of $A$. It is desirable to find some effective procedure to decide when two Bratteli diagrams represent isomorphic AF algebras. Our result can be interpreted as a limitation on the search of such procedures.

Let $\mathrm{BD}$ be the space of all Bratteli diagrams for AF algebras. Using the axiomatisation given in [Br72], BD can be viewed as a Borel subset of $2^{\mathbb{N}^{2}} \times\left(2^{\mathbb{N}^{4}}\right)^{\mathbb{N}}$, so it is a standard Borel space. Consider the equivalence relation on BD where Bratteli diagrams $D$ and $D^{\prime}$ are equivalent iff they represent isomorphic AF algebras. For $K \in \mathcal{K}\left(2^{\mathbb{N}}\right)$ we can associate a Bratteli diagram $D(\mathcal{C}(K))$, which can be identified with the (unique) pruned tree whose infinite branches are the elements of $K$. Then the function $\varphi_{3}: \mathcal{K}\left(2^{\mathbb{N}}\right) \rightarrow$ BD defined by $\varphi_{3}(K)=D(\mathcal{C}(K))$ is a Borel reduction. 
In fact, from a Bratteli diagram we can recover in a Borel way the associated AF algebra and then the corresponding dimension group. In any case, we have the following result.

Theorem 11. The relation of equivalence for Bratteli diagrams is Borel complete.

Our results in this section improve some earlier work of Panti ([Pa95]).

\section{Further PROBLEMS}

In the preceding sections we have shown the Borel completeness of various equivalence relations related to the classification of countable Boolean algebras or of separable Boolean spaces. Here let us point out the following questions which seem still open.

Question. Given an arbitrary countable non-superatomic Boolean algebra $B_{0}$, is the isomorphism relation between the pairs $\left(B_{0}, I\right)$ Borel complete, where $I$ is an ideal of $B_{0}$ ?

In case $B_{0}$ is superatomic, the corresponding isomorphism relation between pairs $\left(B_{0}, I\right)$ can be shown to be Borel. Our Theorem 4 established the Borel completeness when $B_{0}=B_{\eta}$. This only allows us to deduce a positive answer in a few other cases, e.g., when $B_{0}$ is a product of $B_{\eta}$ with a superatomic algebra. In general the answer is unknown. One of the simplest algebras one can consider is $D(1+\omega \eta+1)$, which we also denote by $B_{\omega \eta}$. We feel that any technique that can penetrate this case should be applicable to give a full answer to the above question.

Question. Given an arbitrary countable non-superatomic Boolean algebra $B_{0}$, is the isomorphism relation between the pairs $\left(B_{0}, \varphi\right)$ Borel complete, where $\varphi$ is an automorphism of $B_{0}$ ?

This question is in the same spirit with the preceding one. Again, in case $B_{0}$ is superatomic, the corresponding relation is Borel (cf. Ga98]). Similarly, the case $B_{0}=B_{\omega \eta}$ has not been worked out.

Next let us turn to the Vaught conjecture for Boolean algebras. Our Theorem 2 in section 4 can be viewed as an ultimate solution to the Vaught conjecture for Boolean algebras in which the concept of cardinality is replaced by that of "definable cardinality" (as developed in [BK96]). However, there is another infinitary version of the Vaught conjecture for which we do not know the answer, even in the ordinary sense of cardinality.

Question. Let $\mathcal{L}$ be the language of Boolean algebras. Let $\psi \in \mathcal{L}_{\omega_{1} \omega}$ be a sentence. Is it true that $\operatorname{Mod}\left(T_{\mathrm{BA}} \cup\{\psi\}\right)$ has either countably many or $2^{\aleph_{0}}$ many countable models up to isomorphism?

In the framework of the Borel reducibility hierarchy, the traditional cardinality $2^{\aleph_{0}}$ corresponds to a big family of definable cardinalities, some Borel (meaning it is a Borel equivalence relation) and some non-Borel. A natural successor to the above question is then to further understand the definable cardinalities of the classes of models. In the following we formulate a question of this flavor.

Question. Let $\mathcal{L}$ be the language of Boolean algebras. Let $E$ be a Borel equivalence relation which is the orbit equivalence relation of a Borel $S_{\infty}$-action. Is it true that there is always a sentence $\psi \in \mathcal{L}_{\omega_{1} \omega}$ such that $E$ is Borel equivalent to the isomorphism relation on $\operatorname{Mod}\left(T_{\mathrm{BA}} \cup\{\psi\}\right)$ ? 
In [Ga98] we already developed some techniques to answer this type of question. In fact, it was shown that the analogous question about countable linear orderings has a positive answer. The method used there was to give a detailed analysis of the reduction from graphs to linear orderings in terms of Scott rank preservation. (So, in particular, the answer should not depend on the answer to the infinitary version of the Vaught conjecture.) Here the obstacle is that the reduction we gave in section 3 is not literally a reduction to Boolean algebras, hence making it difficult to figure out whether the reduction preserves the Scott ranks of the models.

Finally let us emphasize that our results in this paper do not in any sense conclude the study of the isomorphism types of countable Boolean algebras. In particular, our approach does not seem to help in understanding the structure of the monoid of all isomorphism types of countable Boolean algebras (see [Pi89]). It would be interesting if some connection with this aspect can be found.

\section{ACKnowledgement}

The work presented in this paper was done under the guidance of Alekos Kechris. Discussions with Greg Hjorth, Slawek Solecki, Alessandro Andretta, Alberto Marcone, Simon Thomas, Edward Effros and Giovanni Panti were of great value. To them we express our heartfelt appreciation.

\section{REFERENCES}

[An58] R. D. Anderson, The algebraic simplicity of certain groups of homeomorphisms, American Journal of Mathematics 80 (1958), 955-963. MR 20:4607

[Ba75] J. Barwise, Admissible Sets and Structures: An Approach to Definability Theory, Perspectives in Mathematical Logic, Springer-Verlag, Berlin, 1975. MR 54:12519

[BK96] H. Becker and A. S. Kechris, The Descriptive Set Theory of Polish Group Actions, London Mathematical Society Lecture Notes Series 232, Cambridge University Press, 1996. MR 98d:54068

[B198] B. Blackadar, K-theory for Operator Algebras, Second Edition, Cambridge University Press, 1998. MR 99g:46104

[Br72] O. Bratteli, Inductive limits of finite dimensional $C^{*}$-algebras, Transactions of the American Mathematical Society 171 (1972), 195-234. MR 47:844

[FS89] H. Friedman and L. Stanley, A Borel reducibility theory for classes of countable structures, The Journal of Symbolic Logic 54 (1989), 894-914. MR 91f:03062

[Ga98] S. Gao, The isomorphism relation between countable models and definable equivalence relations, PhD dissertation, UCLA, 1998.

[Go97] S. S. Goncharov, Countable Boolean Algebras and Decidability, Consultants Bureau, 1997. MR 98h:03044b

[Ha76] W. Hanf, Representing real numbers in denumerable Boolean algebras, Fundamenta Mathematicae 91 (1976), 167-170. MR 54:7252

[Hj98] G. Hjorth, Classification and Orbit Equivalence Relations, Mathematical Surveys and Monographs, 75, Amer. Math. Soc., Providence, RI, 2000. CMP 2000:05

[HK95] G. Hjorth and A. S. Kechris, Analytic equivalence relations and Ulm type classifications, The Journal of Symbolic Logic 60 (1995), 1273-1300. MR 96m:54068

[Ho93] W. Hodges, Model Theory, Cambridge University Press, 1993. MR 94e:03002

[Iv91] P. Iverson, The number of countable isomorphism types of the theory of Boolean algebras, Colloquium Mathematicum 62 (2) (1991), 181-187. MR 93a:03038

[Kec95] A. S. Kechris, Classical Descriptive Set Theory, Springer-Verlag, 1995. MR 96e:03057

[Kec98] A. S. Kechris, The descriptive classification of some classes of $C^{*}$-algebras, Proceedings of the Sixth Asian Logic Conference (1998), 121-149.

[Ket78] J. Ketonen, The structure of countable Boolean algebras, Annals of Mathematics 108 (1978), 41-89. MR 58:10647

[Ko89] S. Koppelberg, Handbook of Boolean Algebras, vol. 1 (J.D. Monk ed.), North-Holland, 1989. MR 90k:06002 
[Mu90] G. J. Murphy, $C^{*}$-algebras and Operator Theory, Academic Press, 1990. MR 91m:46084

[Na72] M. A. Naimark, Normed Algebras, Wolters-Noordhoff, 1972. MR 55:11042

[Pa95] G. Panti, La logica infinito-valente di Eukasiewicz, PhD dissertation, Università degli studi di Siena, 1995.

[Pi89] R. S. Pierce, Countable Boolean algebras, in Handbook of Boolean Algebras (J. D. Monk and R. Bonnet eds.), Elsevier Science Publishers, 1989, 775-876. CMP 21:10

[vM89] J. van Mill, Infinite Dimensional Topology. Prerequisites and Introduction, Elsevier Science Publishers, 1989. MR 90a:57025

Department of Mathematics, California Institute of Technology, Pasadena, CaliFORNIA 91125

E-mail address: camerlo@its.caltech.edu

Department of Mathematics, California Institute of Technology, Pasadena, CaliFORNIA 91125

E-mail address: sugao@its.caltech.edu 